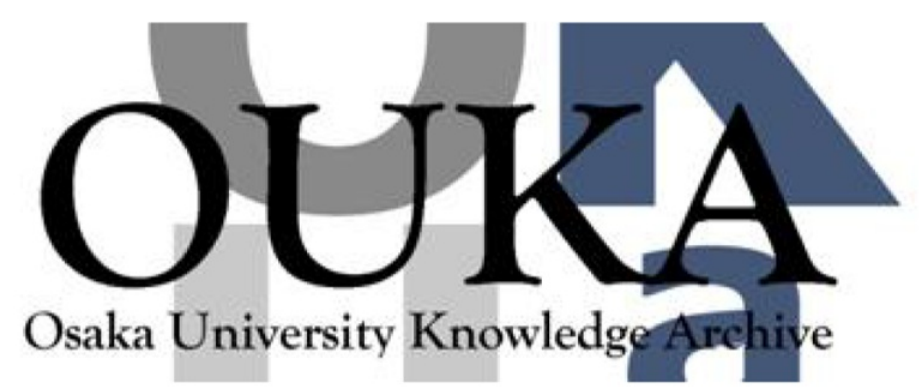

\begin{tabular}{|c|c|}
\hline Title & Systematics of fusioin-fission time scales \\
\hline Author (s) & Hinde, D. J. ; Ogata, H.; Tanaka, M. et al. \\
\hline Citation & Physical Review C. 39(6) p.2268-p. 2284 \\
\hline Issue Date & $1989-06$ \\
\hline oaire:version & VoR \\
\hline URL & https://hdl. handle. net/11094/23133 \\
\hline rights & $\begin{array}{l}\text { Hinde, D. J. , Ogata, H. 'Tanaka, M. , } \\
\text { Shimoda, T. 'Takahashi, N. ' Shinohara, A. } \\
\text { Wakamatsu, S. ' Katori, K. Okamura, H., } \\
\text { Physical Review C, 39, 2268-2284, 1989 } \\
\text { "Copyright (1989) by the American Physical } \\
\text { Society." }\end{array}$ \\
\hline Note & \\
\hline
\end{tabular}

Osaka University Knowledge Archive : OUKA

https://ir. Library. osaka-u. ac. jp/

0saka University 


\title{
Systematics of fusion-fission time scales
}

\author{
D. J. Hinde* and H. Ogata \\ Research Centre for Nuclear Physics, Osaka University, Mihogaoka, Ibaraki, Osaka 567, Japan \\ M. Tanaka \\ Kobe Tokiwa Junior College, Nagata, Kobe 653, Japan \\ T. Shimoda and N. Takahashi \\ College of General Education, Osaka University, Toyonaka, Osaka 560, Japan \\ A. Shinohara ${ }^{\dagger}$ and S. Wakamatsu \\ Department of Chemistry, Faculty of Science, Osaka University, Toyonaka, Osaka 560, Japan \\ K. Katori \\ Laboratory of Nuclear Studies, Faculty of Science, Osaka University, Toyonaka, Osaka 560, Japan \\ H. Okamura \\ Department of Physics, Kyoto University, Kyoto 606, Japan \\ (Received 15 November 1988)
}

\begin{abstract}
New pre-scission neutron multiplicity $\left(\nu_{\text {pre }}\right)$ data for ${ }^{7} \mathrm{Li}-,{ }^{16} \mathrm{O}$-, and ${ }^{20} \mathrm{Ne}$-induced fission are presented, spanning a fissility range from 0.60 to 0.85 . Fission time scales have been deduced for two extreme assumptions regarding the mean excitation energy during fission. It is found for fusion-fission reactions that the fission time scale is independent of fissility, within a factor 1.5. A comparison of time scales deduced from $v_{\text {pre }}$ measurements for fast-fission with quasifission timescales deduced from the rotation angle of the composite system allows a minimum fusion-fission time scale of $30 \times 10^{-21} \mathrm{~s}$ to be determined. For the most fissile system, fast-fission (fission without barrier) is shown to be up to three times faster than fusion-fission. Using a model to interpret the fusion-fission time scales, it is concluded that motion in the fission direction is strongly overdamped. This means that the dynamics of fission are dominated by a slow diffusion towards scission, and not by the potential energy surface. It is shown that such a picture can explain the observed lack of dependence of the fission time scale on fissility.
\end{abstract}

\section{INTRODUCTION}

Elucidation of the precise nature and magnitude of nuclear viscosity remains one of the major problems as yet unsolved in nuclear physics, although substantial progress is being made, both experimentally and theoretically. The unique properties of the nucleus (small number of constituent nucleons, with a long mean free path, at least at temperatures where Pauli blocking is effective) mean that nuclear collisions with the nuclear surface, or nucleon-nucleon collisions in the nuclear surface region are likely to be the main contributors to nuclear viscosity. ${ }^{1}$ Because of these facts, nuclear viscosity should show novel features and complexity not seen in classical macroscopic systems.

Different theoretical approaches to modeling viscosity and dynamics, such as time dependent Hartree-Fock, ${ }^{2}$ wall and window pure one-body viscosity, ${ }^{3}$ macroscopic two-body viscosity, ${ }^{4}$ the surface friction model, ${ }^{5}$ and dissipative diabatic dynamics ${ }^{6}$ show different success in describing different manifestations of nuclear viscosity such as the properties of giant resonances, fusion and fission.
For fission, attempts have been made to gain information on nuclear viscosity from the dependence of the total kinetic energy (TKE) on the compound nucleus fissility. Although several studies have been made comparing the different one- and two-body dissipation mechanisms, ${ }^{7}$ both have given acceptable fits to the experimental systematics. The variation of TKE with excitation energy is expected to be quite different for different dissipation mechanisms, ${ }^{8}$ but it has been recognized ${ }^{9}$ that prescission neutron emission will reduce the possible variation in excitation energy at and near the saddle point to a rather small range, and thus any variation in TKE resulting from a change in viscosity with temperature should also be small. It may not be possible to determine the mechanism and strength of nuclear viscosity from TKE properties alone. Recently, measurements of evaporated charged particle ${ }^{10}$ and neutron multiplicities ${ }^{9,11-20}$ emitted during the fission process have been used as a "clock" to allow estimation of the dynamical fission time scale. This quantity is critically dependent on the magnitude of nuclear viscosity. It may be argued that these measurements represent the clearest and most dramatic evidence of the effects of nuclear viscosity in fission. 
After the formation of a thermally equilibrated compound nucleus in a fusion reaction, in general it will decay by fission or particle evaporation. For heavy nuclei, neutron emission is dominant, and the competition between fission and neutron evaporation describes the decay possibilities rather well. This is expressed in terms of the widths for fission $\Gamma_{f}$ and neutron evaporation $\Gamma_{n}$. The probability of fission from a given nucleus $i$ in the decay chain is given by

$$
P_{f}^{i}=\Gamma_{f}^{i} / \Gamma_{\text {tot }}^{i} \text { where } \Gamma_{\text {tot }}^{i}=\Gamma_{f}^{i}+\Gamma_{n}^{i} .
$$

In the transition state picture of fission, the nucleus is assumed to be committed to fission if it reaches the saddlepoint configuration. The fission width is thus dependent on the probability of finding the nucleus in this configuration, which in the statistical model is simply proportional to the level density at the saddle point. The probability for evaporation of a neutron is similarly proportional to the level density in the daughter nucleus. Thus in this static picture, fission decay and neutron evaporation compete as soon as the thermally equilibrated compound nucleus is formed, and processes occurring beyond the saddle point are ignored. In this picture, fission may occur after the emission of several neutrons, which are called pre-fission neutrons. The mean multiplicity of such neutrons for a compound nucleus with angular momentum quantum number $J$ is given by

$$
v_{\mathrm{pre}}^{J}=\sum_{i}(i-1) P_{f}^{i} / \sum_{i} P_{f}^{i},
$$

where $i$ is the index representing the steps in the neutron evaporation chain, $i=1$ being called first-chance fission, $i=2$ second-chance, etc. The value of $v_{\text {pre }}$ which should be compared with experimental measurements is given by

$$
v_{\mathrm{pre}}=\sum_{J} v_{\mathrm{pre}}^{J} \sigma_{J} / \sum_{J} \sigma_{J},
$$

where $\sigma_{J}$ is the partial fusion cross section for a given angular momentum.

The experimental pre-fission neutron multiplicities $v_{\text {pre }}$ are determined by measuring the angular correlation of neutrons in coincidence with fission fragments traveling in a known direction with known viscosity. Neutrons emitted from the fission fragments will be focused in the fragment direction, and using the neutron energy spectra, the angular correlation can be decomposed into the prescission and post-scission components. Such measurements have shown that at low excitation energy and fissility, agreement with the statistical model calculations using standard parameters can be obtained, ${ }^{12,16,19}$ but at high excitation energy and/or fissility, the experimental yields exceed those calculated manyfold. ${ }^{9-11,13-18,20}$ This can be explained qualitatively in quite general terms. In the statistical model picture described above, the time scale for fission at a certain step $i$ in the evaporation chain can be defined as $\tau_{\text {stat }}=\hbar / \Gamma_{\text {tot }}^{i}$. Where $\Gamma_{\text {tot }}^{i}$ is large, due to high excitation energy and/or fissility, this time scale can become very short $\left(10^{-22} \mathrm{~s}\right.$ or less). It is not realistic to expect a complex collective phenomenon such as fission to occur on such a short time scale. Indeed, classical hydrodynamical calculations for a nucleus with a fission barrier which has been reduced to zero, and with no viscosity, ${ }^{21}$ show that it takes several $10^{-21} \mathrm{~s}$ for the nucleus to move from the saddle configuration to the scission configuration. For such a system, the statistical model pre-fission multiplicity is strictly zero, since the equilibrium and saddle-point deformations coincide. During the collective motion to the scission point, neutrons will be evaporated if energetically possible, and would be experimentally identified as pre-fission, or more correctly, pre-scission neutrons. In this macroscopic picture, the effect of viscosity is to slow the motion to scission. A longer saddle to scission time will result in a higher pre-scission neutron multiplicity, thus the latter quantity can be used to gain information on nuclear viscosity in a rather direct way. For nuclei with a fission barrier, a further effect of viscosity is to delay the onset of fission, due to the time delay in establishing the equilibrium population at the saddle point. ${ }^{22-25}$ This will also result in the emission of excess neutrons. The sum of the time delay before the onset of fission and the saddle to scission transit time, both of which depend on viscosity, can be described as the dynamical fission time scale. $\tau_{\text {stat }}$ also depends on viscosity (see Sec. IV D), as well as on the fission barrier height, level densities, and other statistical model parameters, but its value is constrained by the fission probability, which in many cases has been measured, or at least can be estimated from systematics. It is thus essential to make the distinction between the statistical model fission time scale, which in practice must be adjusted to be independent of viscosity, and the dynamical fission time scale, which is not constrained by the fission probability, but will determine the pre-scission neutron multiplicity if it is longer than $\tau_{\text {stat }}$. The use of the term fission time scale in this work refers to the dynamical time scale.

Experimentally, evidence for long fission time scales comes from fission angular distributions, whose isotropic yield in $\theta$ shows that typically the nucleus probably rotates several times before scission occurs. Pre-scission neutron measurements also indicate ${ }^{11,15,16}$ long fission time scales of $\sim$ several $10^{-20} \mathrm{~s}$. To attempt to make quantitative deductions of fission time scales and systematics from $v_{\text {pre }}$ values, it is necessary to address several problems. It must be clear whether the fission events observed result from the pure fusion-fission reaction, or from a mixture of reaction types. In fusionfission, the projectile is completely absorbed by the target, and it is assumed that the resulting compound nucleus reaches its equilibrium (near spherical) deformation before proceeding in the fission direction. In order for this to occur, it is necessary for the system to have a fission barrier. If the angular momentum brought in is so high that the fission barrier is reduced to zero, then it is found that the fission mass width increases. This has been interpreted ${ }^{26}$ as evidence that the fission occurs on a faster time scale than fusion-fission. Such fissionwithout-barrier is generally called fast-fission, and although in absolute terms it may not be considered as "fast," it seems reasonable that it should be faster than fusion-fission. A further class of fission has been observed $^{8,27}$ in reactions between nuclei with a large 
Coulomb energy $\left(Z_{1} Z_{2} \geq 1600\right)$. Although possessing a fission barrier, these systems also show evidence for fission occurring on a fast time scale, namely the correlation of the mean fission fragment mass with detection angle, and very broad mass distributions. It has been suggested $^{8}$ that due to the high Coulomb repulsion the fission trajectory does not pass inside the true (unconditional) fission barrier, in other words true fusion does not occur. This process has been called quasifission. Conceptually, the difference between fast-fission and quasifission is not very great. Although experimentally they seem to show rather different properties (for example the widths of the fission fragment mass distributions), care must be taken in making comparisons between reactions involving systems with different fissility and Coulomb energy.

To investigate these different classes of fission and compare the reaction times of each would be interesting, however the degree of overlap between them must be considered, and reactions must be chosen carefully to avoid confusion.

Considering first the fusion-fission reaction, the existing $v_{\text {pre }}$ data for fission induced by heavy ions can be divided into two categories, those measured at low energies, $, 12,16,18,19$ up to $\sim 1.5$ times the fusion barrier height, and those at higher energies. The former are not so sensitive to the fission time scale, but give valuable information on the statistical model parameters (except for the most fissile systems which would be classed with the high energy data), while the latter are quite insensitive to the statistical model parameters, and thus can give fission time scales with less uncertainty, but may include contributions from fast-fission. The latter data comprise the following.

(1) Measurements for Ir compound nuclei ${ }^{10,13,15}$ using ${ }^{12} \mathrm{C},{ }^{20} \mathrm{Ne}$, and ${ }^{40} \mathrm{Ar}$ beams; only the reaction induced by ${ }^{12} \mathrm{C}$ should be free of fast-fission, but there is some uncertainty in the proportion of complete and incomplete fusion, which could cause uncertainty in extracting a time scale.

(2) Measurements for the ${ }^{170} \mathrm{Yb}$ compound system ${ }^{11}$ induced by $192 \mathrm{MeV}{ }^{12} \mathrm{C}$ and $176,239 \mathrm{MeV}^{20} \mathrm{Ne}$ projectiles; only the highest beam energy should result in fastfission and the total neutron multiplicities for the other reactions indicate essentially complete fusion preceding fission. ${ }^{16}$

(3) Measurements for the ${ }^{158} \mathrm{Er}$ system ${ }^{17}$ induced by ${ }^{16} \mathrm{O},{ }^{24} \mathrm{Mg},{ }^{32} \mathrm{~S}$ and ${ }^{50} \mathrm{Ti}$; it has already been shown ${ }^{20}$ that these data give $v_{\text {tot }}$ results lower than expected from energy balance, and $v_{\text {pre }}$ results different from those for the systems mentioned above, which indicates a discrepancy in the results.

The low energy data give valuable information on time scales for the more fissile systems, but for compound nuclei lighter than $A \sim 225$ are rather sensitive to the statistical model parameters used.

Thus although there have been rather a lot of measurements, not many allow an unambiguous determination of the fusion-fission time scale (although all will be very useful when further understanding of all aspects of fusion and fission is to be obtained).

In view of this fact, a series of measurements for fusion-fission induced by ${ }^{16} \mathrm{O}$ and ${ }^{20} \mathrm{Ne}$ beams have been made in the high energy regime, but at energies where fast-fission should not occur, for reactions which complement those studied at low energies. These measurements extend the rather limited data currently available. Measurements using the ${ }^{20} \mathrm{Ne}$ beam were also made at higher bombarding energies, where there is a probability of some component of fission-without-barrier (fast-fission), to gain some information on the relative time scales of these two processes. For the most fissile system, at the highest bombarding energy, the measurement was made as a function of the fission mass split and total kinetic energy, to see whether separate components due to fast-fission and fusion-fission could be identified. Measurements were also carried out for fission induced by a ${ }^{7} \mathrm{Li}$ beam, where the angular momentum brought in is lower than for the heavier beams. Si detector telescopes were included in the setup to allow estimation of the yield of evaporated charged particles, which could be significant for the lightest and most neutron deficient system.

\section{EXPERIMENTAL METHOD}

The experiment was carried out at the Research Centre for Nuclear Physics (RCNP), Osaka University. Beams of $178 \mathrm{MeV}{ }^{16} \mathrm{O}, 149$ and $215 \mathrm{MeV}{ }^{20} \mathrm{Ne}$, and $148 \mathrm{MeV}$ ${ }^{7} \mathrm{Li}$ extracted from the cyclotron were incident on targets of ${ }^{142} \mathrm{Nd},{ }^{168} \mathrm{Er},{ }^{181} \mathrm{Ta},{ }^{197} \mathrm{Au},{ }^{209} \mathrm{Bi}$, and ${ }^{232} \mathrm{Th}$. Details of the reactions, target thicknesses and mean energies in the targets are given in Table $I$. The reactions were chosen to complement, as far as was possible, the measurements made previously ${ }^{9,16,19}$ at lower bombarding energies. The experimental configuration is shown in Fig. 1. Fission fragments were detected in the Si detectors $f_{1-3}$, located $10 \mathrm{~cm}$ from the target and with $13 \mathrm{msr}$ solid angle. The Si detector telescopes $\alpha_{1-2}$, used to determine the light charged particle multiplicities, were at $7 \mathrm{~cm}$, while the large area $\mathrm{Si}$ detectors $F_{1-3}$, with active diameter $2.2 \mathrm{~cm}$, were typically $6 \mathrm{~cm}$ from the target. All detectors were in the plane perpendicular to the beam except $F_{1-3}$,

TABLE I. Properties of beams and targets used in this work. Target thickness (I) are those used in the neutron measurements, while set (II) were used for the measurements of the fission fragment mass distributions and for the ${ }^{20} \mathrm{Ne}+{ }^{232} \mathrm{Th}$ neutron measurement.

\begin{tabular}{lcccccc}
\hline \hline \multicolumn{1}{c}{ Target } & ${ }^{142} \mathrm{Nd}^{\mathrm{a}}$ & ${ }^{168} \mathrm{Er}$ & ${ }^{181} \mathrm{Ta}$ & ${ }^{197} \mathrm{Au}$ & ${ }^{209} \mathrm{Bi}$ & ${ }^{232} \mathrm{Th}^{\mathrm{b}}$ \\
\hline Thickness (I) & 1.0 & 1.10 & 1.0 & 0.78 & 1.4 & 0.68 \\
$\left(\mathrm{mg} / \mathrm{cm}^{2}\right)(\mathrm{II})$ & & & 0.35 & & 0.24 & 0.20 \\
Beam & ${ }^{16} \mathrm{O}$ & ${ }^{20} \mathrm{Ne}$ & ${ }^{20} \mathrm{Ne}$ & ${ }^{16} \mathrm{O}$ & ${ }^{20} \mathrm{Ne}$ & ${ }^{20} \mathrm{Ne}$ \\
$E_{\text {lab }}{ }^{20}$ & 178 & 148 & 148 & 178 & 148 & \\
$(\mathrm{MeV})$ & & 214 & 214 & & 214 & 215 \\
Beam & & & ${ }^{7} \mathrm{Li}$ & ${ }^{7} \mathrm{Li}$ & ${ }^{7} \mathrm{Li}$ & ${ }^{7} \mathrm{Li}$ \\
$E_{\text {lab }}$ & & & 148 & 147 & 147 & 148 \\
$(\mathrm{MeV})$ & & & & & & \\
\hline \hline
\end{tabular}

${ }^{\text {a}}$ Oxide target.

${ }^{b}$ Fluoride target.

${ }^{\mathrm{c}}$ Mean energy in the target for the $v$ measurements. 


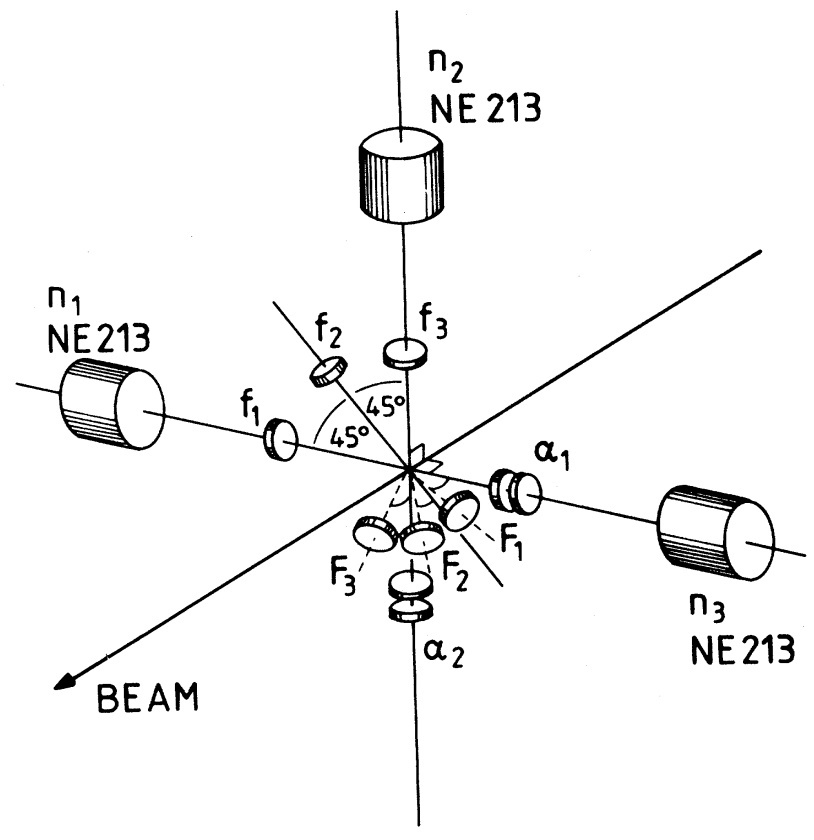

FIG. 1. Schematic diagram of the present experimental configuration showing the neutron detectors $n_{1-3}$, principal fission detectors $f_{1-3}$, and alpha-particle telescopes $\alpha_{1-2}$, all in the plane perpendicular to the beam. The complementary fission fragment detectors $F_{1-3}$ are located to select fission following complete fusion. See text for further details.

which were positioned to intercept the fragments complementary to those detected in $f_{1-3}$. These detectors were moved as the target and beam were changed so as to coincide (to within $\pm 2 \mathrm{deg}$ ) with the expected ${ }^{28}$ peak in the folding angle distribution, corresponding to maximum momentum transfer. All the Si detectors were mounted in a cylindrical Al scattering chamber of wall thickness $0.5 \mathrm{~cm}$ whose axis coincided with that of the beam. NE-213 neutron detectors $(12.7 \mathrm{~cm} \times 12.7 \mathrm{~cm}$ with $27 \mathrm{msr}$ solid angle) were placed coaxially with detectors $f_{1}, f_{3}$, and $\alpha_{1}$.

The data collection was triggered by an event (usually a fission fragment) in one of the $f$ detectors or in the front elements of the $\alpha$ telescopes. This opened the gate for the $F$ detectors. If a signal was present in one of the back elements of the $\alpha$ telescopes, or in one of the neutron detectors, all parameters were stored, otherwise the singles events were prescaled by 20 before storage. Thus the neutron and charged particle multiplicities could in principle be measured simultaneously both for all fission, and for fission following maximum momentum transfer. In the former mode, fission fragments were identified only by their energy, but comparison with the energy spectrum for coincident events showed that this was quite adequate. The data presented will however be almost exclusively for fission following maximum momentum transfer (see Sec. IV B). The time-of-flight measurement for the neutrons was started by the arrival times of the fission fragments at the $f$ detectors; after correcting for the dependence of flight time on fragment energy, the resolution [measured by the full width at half maximum (FWHM) of the $\gamma$-ray peak] was $1.8 \mathrm{~ns}$. The efficiencies of the neutron detectors were measured as a function of neutron energy (velocity) using a ${ }^{252} \mathrm{Cf}$ source mounted in a $2 \pi$ multiwire proportional counter ${ }^{19}$ before, during, and after the series of measurements. The neutron detector energy thresholds were set low so that their possible fluctuation would have a negligible effect on the efficiency at energies where the neutron yield was high. During the bombardments, the contribution from random coincidences was determined by recording the spectrum from the next beam burst. This was typically $1 \%$, reaching a maximum value of $5 \%$, and was corrected for. All the neutron time signals were routed into one time-toamplitude converter (TAC), which meant that the relative yields from each neutron detector should be almost error free, since the same dead times would apply to all. This procedure did have the disadvantage that multiple detector firings caused pileup in the TAC, however, these events were recorded and identifiable. The magnitude of this effect was only 5-10\%, and was corrected for.

For the reaction of $215 \mathrm{MeV}{ }^{20} \mathrm{Ne}$ on ${ }^{232} \mathrm{Th}$, the neutron multiplicity was measured as a function of the fission mass split and total kinetic energy (TKE). To determine these quantities, an energy and pulse-height defect (PHD) calibration was made for all the Si detectors, using a ${ }^{252} \mathrm{Cf}$ source and pulser. The PHD measured for the fission fragments from ${ }^{252} \mathrm{Cf}$ was fitted using the procedure of Kaufman et al. ${ }^{29}$ Calibrations were done before, during and after the measurement. The $F$ detectors showed an increase in PHD with increased exposure. The mass and TKE widths were measured for thin ${ }^{181} \mathrm{Ta}$ and ${ }^{209} \mathrm{Bi}$ targets as well as for the thin ${ }^{232} \mathrm{Th}$ target to compare the results with systematics, and give some check on the reliability of the system.

\section{DATA INTERPRETATION}

Transformation of the raw data to obtain information on fission time scales and nuclear viscosity involves several steps. In this section the transformation procedure will be discussed, emphasizing the possible errors or uncertainties which may accompany each step, in particular as they relate to this experiment.

\section{A. Identification of fission fragments}

Identification of fission fragments was not difficult as they have rather distinctive properties for the reactions studied here. However, to accurately measure the fission mass split and TKE using Si detectors is rather difficult in a reaction giving considerable excitation energy and recoil velocity to the compound system. Fortunately in this measurement very good resolution was not required partly due to the problem of collecting sufficient fissionneutron coincidences and partly because no rapid change of properties with mass split or TKE is expected. Thus, a rather coarse division of the mass and TKE is acceptable.

The mass split was determined from the ratio of the fragment kinetic energies, corrected event-by-event for the reaction kinematics and detector PHD in an iterative procedure, which also gave the TKE. The mass and 
TKE resolution was expected to be limited by the effects of the $f$ detector solid angle and the effects of neutron evaporation, however during the experiment some problem of electron noise was observed, probably caused by poor vacuum in the beamline. As will be discussed later, these two factors resulted in a mass resolution probably worse than 10 mass units.

The above discussion only related to the measurements as a function of mass split and TKE. For all the other measurements, only the identification of fission fragments was required.

\section{B. Determination of neutron multiplicities}

To deduce the correct pre- and post-scission neutron multiplicities from the measured fission-neutron angular correlations, several experimental effects must be accounted for.

\section{Neutron detector efficiencies}

The neutron detector efficiencies must be known. In this work they were measured in the experimental configuration. A $2 \pi$ multiwire proportional counter with a ${ }^{252} \mathrm{Cf}$ source as one of the cathode planes was placed in the target position to give a start signal for measuring the neutron spectrum. This was divided by the well-known ${ }^{30}$ neutron singles spectrum from ${ }^{252} \mathrm{Cf}$ to give the detector efficiencies. Thus individual properties of each detector, such as light transmission efficiency, resolution, and scattering of neutrons into the allowed region in the recoil energy versus true energy matrix are accounted for. Furthermore, in conjunction with a low energy threshold, measurement of the efficiency allowed reliable data to be taken down to low neutron energy $(\sim 0.5 \mathrm{MeV})$. This avoids possible errors caused by extrapolation to these energies, and also constrains the fit parameters, giving less uncertainty in the deduced multiplicities.

\section{Neutron energy spectrum}

A realistic parametrization of the neutron spectral shapes should in principle be used (although in practice it has been found that the extracted multiplicities are not very sensitive to this point). Nevertheless, in this work some effort has been made in this direction. A simple neutron evaporation cascade calculation was built into the fitting program. For a given step, the neutron energy spectrum was taken to have the form $E_{n} / T^{2} \exp \left(E_{n} / T\right)$, where $T$ was calculated from the excitation energy using a level density parameter $a_{n}=A / 10$. Isotropic emission in the rest frame of the source was assumed. ${ }^{15}$ From a given starting energy, the mean temperature at each step in the cascade was determined from the energy removed in previous steps. By summing the contributions from all steps, the final spectral shape was determined. A smooth transition in excitation energy between the pre-scission and post-scission spectra was built in, to simulate the effect of a distribution of pre-scission lifetimes. In the fitting procedure, the pre-scission and post-scission multiplicities were independently varied, as were the starting and stopping energies of the pre-scission cascade (from the fission $Q$ value the latter fixed the starting energy of the post-scission cascade), which is equivalent to the variation of the temperature parameters in Ref. 17. The $\chi^{2}$ surface was mapped out, and best fitting values of the pre-scission and post-scission multiplicities, with standard statistical uncertainties were determined.

\section{Fission fragment velocities}

The mean value and width of the fission fragment velocity and angle distributions for both fragments should be correctly modeled in the fitting procedure, since the whole experimental method is based on the focusing of post-scission neutrons due to the fission fragment velocity. In this work the velocities could not be measured directly, because the time width of the beam pulses was too great. Thus the mean value was taken from the Vio$\mathrm{la}^{31}$ TKE systematics. The TKE values which were fitted in that compilation were corrected to represent the TKE before post-scission emission. However at that time it was assumed that no pre-scission neutrons were emitted. It is now known that this assumption is not correct, thus the TKE values fitted were slightly too high, whether they were derived from measurements of fission fragment energies or velocities. Consequently, in order to calculate fission fragment velocities from the systematics, it should be assumed that there are no pre-scission neutrons (the mean fission-fragment mass at scission should be taken as half the compound nucleus mass) although this is known to be incorrect. The dependence of the calculated TKE with fissility also can affect the results for nuclei removed from the $A \sim 200$ region. For example, using the 1966 $\mathrm{fit}^{32}$ gives a $3 \%$ increase in $v_{\text {pre }}$ for the ${ }^{158} \mathrm{Er}$ system compared to the $1985 \mathrm{fit}^{31}$ which is expected to be more accurate; the latter was used in this work. The width of the fragment velocity distributions was calculated taking the mass and TKE distributions from systematics and folding in the $f$ and $F$ detector angular acceptances, and allowing an extra spread in angle due to neutron evaporation.

\section{Preequilibrium emission}

The effect of preequilibrium emission must be considered. The experimental configuration used in this work did not allow the extraction of any information on the preequilibrium multiplicity $v_{\mathrm{pe}}$. Since the data were all taken at $90 \mathrm{deg}$, the yield from $v_{\mathrm{pe}}$ should be the same at each neutron detector angle, for the energies used here. ${ }^{15}$ For each reaction studied, the value of $v_{\mathrm{pe}}$ and its spectral shape were obtained from systematics, ${ }^{33,34}$ allowing calculation of the expected spectrum at 90 deg to the beam. It constituted $\leq 5 \%$ of the observed yield. The kinematic focusing was typically such that increasing $v_{\mathrm{pe}}$ by 0.1 caused a reduction in $v_{\text {pre }}$ by 0.07 . Since the values of $v_{\text {pe }}$ ranged from 0.2 to 0.7 for the ${ }^{16} \mathrm{O},{ }^{20} \mathrm{Ne}$ induced reactions, errors in these values will not have a large effect on the quoted values of $v_{\text {pre }}$. Furthermore, as will be described later, the total evaporated multiplicity is used to define the initial thermal excitation energy, so uncertainty in $v_{\text {pe }}$ gives almost no uncertainty in deduced time 
scales. It is thus felt that for these reactions, the lack of new data on $v_{\mathrm{pe}}$ is not a handicap.

\section{Determination of fission time scales}

For a given measurement, the neutrons emitted in excess of the statistical model calculation have been transformed to yield the dynamical fission time scale. This was accomplished essentially as described in Ref. 16 for saddle-to-scission emission. Ignoring charged particle emission, the neutron emission lifetimes are given by

$$
\tau_{n}=\hbar / \Gamma_{n},
$$

where $\Gamma_{n}$ is the neutron emission width, which is essentially determined by the ratio of the level densities after and before neutron emission. This ratio depends on several parameters, through the expression for the level density, here taken to have the form

$$
\rho_{n} \propto\left[(2 J+1) / U^{2}\right] \exp \left\{2 \sqrt{a_{n} U}\right\},
$$

where $a_{n}$ is the level density parameter and $U$ is the thermal excitation energy defined as $U=E_{X}-\left(E_{R}+E_{D}\right)$. Here $E_{X}$ is the total excitation energy above the ground state, $E_{R}$ is the rotational energy, and $E_{D}$ is the deformation energy. Possible uncertainties in these parameters, and their effect on the interpretation of experimental data will now be discussed.

The initial excitation energy at which the compound nucleus was formed is known if the reaction proceeded by complete fusion, otherwise it can be estimated from the deduced momentum transferred, or deduced from the multiplicities of all evaporated particles. Since the former was not measured in this experiment, the latter quantity has been used. From the success of a simple model in describing $v_{\text {tot }}$ values for reactions proceeding by complete fusion, ${ }^{16}$ it can be estimated that excitation energies should be measurable to better than $5 \%$ by this method, as long as the energy removed by charged particles is not neglected. In this work, charged particles were measured, and estimates were made of the charged particle multiplicities. However they were found to make an insignificant correction for all but the lightest and most neutron-deficient system. It must be emphasized that the use of an accurate value of the initial excitation energy is essential in transforming $v_{\text {pre }}$ to a fission time scale, and at bombarding energies per nucleon $(E / A)>10 \mathrm{MeV}$, complete fusion cannot be assumed. It should be noted however that the fission process favors the complete fusion reaction for lighter, less fissile compound nuclei, which will only fission at high angular momentum. From this initial value of the excitation energy, the distribution of $E_{X}$ at the saddle point was determined from the statistical model calculation. If the statistical model value of $v_{\text {pre }}$ is low, $E_{X}$ is high, and vice versa. Thus the values of $\tau_{n}$ for subsequent neutron emission are short or long, respectively, and so a considerable degree of anticorrelation of the statistical model and dynamical neutron multiplicities is found if a fixed dynamical time scale is taken. Thus the conclusions are not very sensitive to the value of $v_{\text {pre }}$ calculated using the statistical model as long as the difference between the statistical model value of $v_{\text {pre }}$ and the measured value is not too small (see Refs. 9 and 19).

The values of $E_{R}$ and $E_{D}$ are tied together, in that the nucleus on average tends to minimize the sum of the two, and thus maximize the level density. In reality they are time dependent, as the nucleus moves from the equilibrium deformation to the scission configuration, however at this stage of our understanding, a fixed value will be used. The fission process itself minimizes the variations in thermal excitation energy, since fission is only probable when the deformation dependence of $E_{R}+E_{D}$ in the fission direction is rather small-typically when the fission barrier is less than $6 \mathrm{MeV}$. Thus the calculations of the rotating liquid drop model (RLDM) in its original $^{35}$ or improved form ${ }^{36}$ allow estimation of a typical value of $U$ for emission from the saddle-point region $\left(U_{\mathrm{sp}}\right)$. This represents the lower limit of the mean excitation energy during fission. For emission during the saddle-to-scission transition, some estimate should be made of the values of $E_{R}+E_{D}$. A configuration of spherical fission fragments gives the lower limit to $E_{R}+E_{D}$, and thus the upper limit to the thermal excitation energy ( $U_{\text {scis }}$ ). Then by taking the average of this value and that at the saddle point, the upper limit to the mean excitation energy during the saddle-to-scission transition is obtained, given by $U_{\mathrm{ssc}}=0.5\left(U_{\mathrm{sp}}+U_{\text {scis }}\right)$. This almost certainly gives an excitation energy that is unrealistically high, but allows comparison with the results of previous analyses where this assumption was made. ${ }^{9,15,16}$

From a consideration of the shape of the potential energy surface, it would be expected that the nucleus should spend most of its time in the equilibrium and saddle regions, however some effect of the fission $Q$ value on the mean excitation energy during fission might be expected, so the data will be analyzed under the two extreme assumptions of saddle-point ( $\mathrm{sp}$ ) emission, and saddle-toscission (ssc) emission. The effect of uncertainties in other parameters must also be considered.

The calculated neutron lifetimes are strongly influenced by the level density parameter $a_{n}$. In this work the value has been taken to be $A / 10$; using $A / 8$ $(A / 12)$ results in deduced fission time scales longer (shorter) by about a factor of 2 . Recent measurements ${ }^{37,38}$ of statistical $\gamma$ rays require a level density parameter of $A / 9$ to fit the energy spectrum, although the uncertainty is still rather large. In principle, the level density parameter can be found from the slope of the measured neutron energy spectrum, however the deduced slope depends on the spectral shape of the preequilibrium neutrons, which must be subtracted off. The analysis of Ref. 17 concludes that $a_{n}=A / 7.5$. Uncertainty in the value of $a_{n}$ determined in this way results from the problem of correctly characterizing the preequilibrium neutron energy spectra and transforming to the laboratory frame; it has been suggested that serious errors may result from the normal procedure of describing the preequilibrium component in terms of only one temperature and one source velocity. ${ }^{39}$ Ideally, $a_{n}$ should be determined in a way which is not dependent on a parametrization of the preequilibrium neutron spectral 
shape, for example by using a reaction where the relative velocity of the projectile and target is low (by using a heavy beam high excitation energy could still be deposited in the compound nucleus). In this case the yield of preequilibrium neutrons should be negligible. In the present measurements no information on the preequilibrium component was obtained, so it was impossible to attempt to determine a value of $a_{n}$ from the neutron energy spectra.

The pre-fission neutron multiplicity calculated in the statistical model picture can be quite large for high excitation energies where the angular momentum corresponds to the point at which the fission barrier is equal to the neutron binding energy. ${ }^{15,16}$ Where the difference between the calculated (statistical model) value and the experimental value of $v_{\text {pre }}$ is less than $\sim 1.5$, the deduced fission time scale is sensitive to the parameters used in the calculation, ${ }^{9}$ which have some uncertainty. For the present results, induced by ${ }^{16} \mathrm{O}$ and ${ }^{20} \mathrm{Ne}$ beams, the angular momentum brought in is sufficient to reduce the sensitivity to small levels, unless gross variations in the statistical model parameters were made.

It will be shown in Sec. IV D that the total fission time scale can be written as the sum of the statistical model time scale, the delay time before the fission rate achieves half its asymptotic value, and the saddle-to-scission time. In the analysis described above, time scales have been deduced for neutron emission after the statistical model neutrons have been emitted, effectively assuming that the delay time is zero. However, for the reactions studied, the statistical model time scale is much smaller than the dynamical time scale, so little error would result if the full pre-scission neutron multiplicity was used to deduce the dynamical time scale, using the initial compound nucleus excitation energy as the starting point for the calculation, rather than the distribution calculated after the statistical model neutrons were emitted (see above). If this were done, the average deformation energy during the whole fission process should be subtracted from the excitation energy when calculating neutron lifetimes, and the time scale extracted would represent the sum of the two dynamical components. For the analysis carried out, the deduced time scales can also be interpreted as the sum of the two dynamical time scale components, as long as the average deformation energy appropriate during the delay time is not very different from that during the saddle-to-scission transition. The two extremes used for the latter deformation energy cover the possible range of the former, so this condition is fulfilled. Thus it is proposed to compare the deduced "saddle" or "saddle-toscission" times with the sum of the two dynamical time scales calculated using a model (see Sec. IV D).

\section{Determination of nuclear viscosity}

In principle, transformation of the measurements to a viscosity should be made in terms of a full reaction trajectory calculation in multiparameter space, from thermal equilibration to scission, including neutron evaporation. In the absence of such extremely difficult calculations, quantitative conclusions regarding the strength of nuclear viscosity are rather difficult to reach, since (as dis- cussed in the Introduction) it is not even clear what form nuclear viscosity takes.

As a first step in interpreting these data, the deduced fission time scales will be presented as a function of compound system mass (fissility) for the different assumptions made in transforming the measured multiplicities to time scales. From these model independent experimental results, the essential features which models of fission dynamics must try to reproduce can be extracted, and then using a model, some information on the essential characteristics of nuclear viscosity for fission at these excitation energies can be deduced.

\section{RESULTS AND DISCUSSION}

The experimental results will be presented and discussed in three sections, namely the data for the ${ }^{16} \mathrm{O}$ and ${ }^{20} \mathrm{Ne}$ induced reactions, the ${ }^{7} \mathrm{Li}$ induced reactions, and finally the mass and TKE dependence for the $215 \mathrm{MeV}$ ${ }^{20} \mathrm{Ne}+{ }^{232} \mathrm{Th}$ reaction. This will be followed by the interpretation in terms of nuclear viscosity.

\section{A. ${ }^{16} \mathrm{O}$ and ${ }^{20} \mathrm{Ne}$ induced reactions}

The experimental results are summarized in Table II, which also shows the maximum angular momentum introduced in the fusion reaction (according to the Bass model ${ }^{40}$ ) and the approximate angular momentum at which the fission barrier becomes zero (according to the rotating finite range model of Sierk $^{36}$ ). From these figures it is clear that the reactions can be divided into two categories, those where fission without barrier (fastfission) is probable and those where it is not.

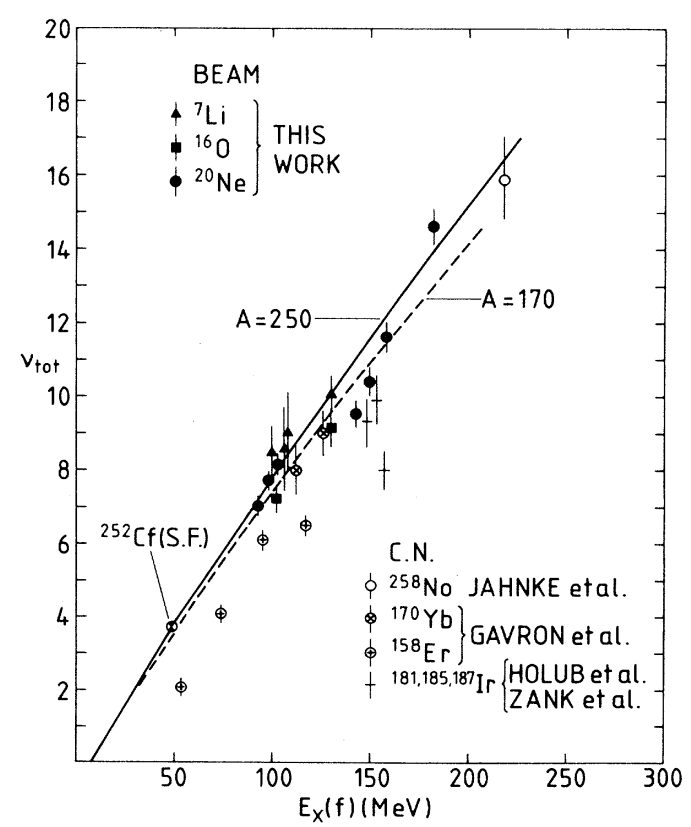

FIG. 2. Total neutron multiplicities for fission, plotted as a function of the total decay energy (see text). The results of a simple calculation using a level density parameter $A / 10$ for fission of nuclei with mass numbers 170 and 250 are shown. 
TABLE II. Properties of the reactions induced by ${ }^{16} \mathrm{O},{ }^{20} \mathrm{Ne}$, together with the neutron multiplicities obtained, and their errors (in parentheses), which include both random errors from the data and spectral decomposition, and also a systematic error in some cases (see text). Any overall systematic error is not included, but from the $v_{\text {tot }}$ results it may be expected to be less than $5 \%$.

\begin{tabular}{|c|c|c|c|c|c|c|c|c|c|c|}
\hline Reaction & $\begin{array}{c}E_{\text {lab }} \\
(\mathrm{MeV})\end{array}$ & $\begin{array}{c}\mathrm{CN} \\
\text { fissility } \\
X \\
\end{array}$ & $l_{E f}=0$ & \begin{tabular}{c}
\multicolumn{2}{c}{ Bass } \\
$l_{\text {crit }} o$ \\
\end{tabular} & $\begin{array}{c}\text { model } \\
\sigma_{\text {fus }}(\mathrm{mb})\end{array}$ & $\begin{array}{l}\text { Fast- } \\
\text { fission }\end{array}$ & $v_{\mathrm{pe}}$ & $\begin{array}{c}\boldsymbol{v}_{\mathrm{pre}} \\
\text { (error) }\end{array}$ & $v_{\text {post }}$ & $v_{\text {tot }}{ }^{\mathrm{a}}$ \\
\hline $\mathrm{O}+\mathrm{Nd}$ & 178 & 0.603 & 78 & 70 & 1450 & NO & 0.70 & $\begin{array}{c}3.95 \\
(0.30)\end{array}$ & $\begin{array}{c}1.45 \\
(0.11)\end{array}$ & $\begin{array}{c}6.84 \\
(0.20)\end{array}$ \\
\hline \multirow[t]{2}{*}{$\mathrm{Ne}+\mathrm{Er}$} & 148 & 0.678 & 78 & 68 & 1335 & NO & 0.25 & $\begin{array}{c}3.53 \\
(0.30)\end{array}$ & $\begin{array}{c}1.74 \\
(0.15)\end{array}$ & $\begin{array}{c}7.00 \\
(0.25)\end{array}$ \\
\hline & 214 & 0.678 & 78 & 99 & 1920 & YES & 0.65 & $\begin{array}{c}5.20 \\
(0.40)\end{array}$ & $\begin{array}{c}2.16 \\
(0.17)\end{array}$ & $\begin{array}{c}9.52 \\
(0.40)\end{array}$ \\
\hline \multirow[t]{2}{*}{$\mathrm{Ne}+\mathrm{Ta}$} & 148 & 0.720 & 74 & 71 & 1410 & NO & 0.25 & $\begin{array}{c}3.80 \\
(0.30)\end{array}$ & $\begin{array}{c}1.95 \\
(0.15)\end{array}$ & $\begin{array}{c}7.70 \\
(0.25)\end{array}$ \\
\hline & 214 & 0.720 & 74 & 99 & 1880 & YES & 0.60 & $\begin{array}{c}5.45 \\
(0.40)\end{array}$ & $\begin{array}{c}2.48 \\
(0.18)\end{array}$ & $\begin{array}{c}10.40 \\
(0.40)\end{array}$ \\
\hline $\mathrm{O}+\mathrm{Au}$ & 178 & 0.751 & 72 & 89 & 2180 & YES $^{\mathrm{b}}$ & 0.60 & $\begin{array}{c}4.85 \\
(0.50)\end{array}$ & $\begin{array}{c}2.15 \\
(0.22)\end{array}$ & $\begin{array}{c}9.15 \\
(0.50)\end{array}$ \\
\hline \multirow[t]{2}{*}{$\mathrm{Ne}+\mathrm{Bi}$} & 148 & 0.801 & 68 & 66 & 1200 & NO & 0.20 & $\begin{array}{c}3.20 \\
(0.40)\end{array}$ & $\begin{array}{c}2.49 \\
(0.31)\end{array}$ & $\begin{array}{c}8.17 \\
(0.30)\end{array}$ \\
\hline & 214 & 0.801 & 68 & 99 & 1850 & YES & 0.55 & $\begin{array}{c}5.12 \\
(0.40)\end{array}$ & $\begin{array}{c}3.22 \\
(0.25)\end{array}$ & $\begin{array}{c}11.55 \\
(0.50)\end{array}$ \\
\hline $\mathrm{Ne}+\mathrm{Th}$ & 215 & 0.854 & 58 & 99 & 1800 & YES & 0.50 & $\begin{array}{c}6.95 \\
(0.40)\end{array}$ & $\begin{array}{c}3.83 \\
(0.22)\end{array}$ & $\begin{array}{c}14.60 \\
(0.50)\end{array}$ \\
\hline
\end{tabular}

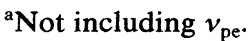

${ }^{\mathrm{b}}$ Incomplete fusion and preequilibrium neutron emission will reduce $l_{\text {crit }}$ so that fast-fission is unlikely for this system (see text).

\section{Total neutron multiplicities}

The total neutron multiplicities can be compared with expectations based on evaporation calculations and energy balance. Since preequilibrium neutrons have a higher kinetic energy than evaporated neutrons, the preequilibrium yield has been excluded from the total multiplicity, to give the total evaporated multiplicity $\left(v_{\text {tot }}\right)$, and correspondingly, the excitation energy has been reduced by 20 $\mathrm{MeV}$ (Ref. 17) for each preequilibrium neutron. The total evaporated neutron multiplicities for both these categories, and those from similar reactions, ${ }^{11,13,15,17,41}$ are shown in Fig. 2 as a function of the total available decay energy $E_{X}(f)$ (see Appendix 1 of Ref. 16) to allow a clear graphical comparison. From the data collected in the two Si detector telescopes, estimates were made of the multiplicities of evaporated charged particles $(Z=1, Z=2){ }^{20}$ These indicate a significant yield only for the lightest and most neutron deficient system where a neutron multiplicity of 0.42 has been added to the value of $v_{\text {tot }}$ plotted for this reaction; ${ }^{20}$ no correction has been applied apart from this case.

Assuming complete fusion followed by evaporation of neutrons and $\gamma$ rays only, a simple ${ }^{16}$ calculation can be made of the expected multiplicities. For a level density parameter of $a_{n}=A / 10$, curves are shown in Fig. 2 for $A=170$ and $A=250$. For the lower values of $E_{X}(f)$, the data agree well with the calculations, however for higher values the measurements fall below the curves, the deficiency depending on the fraction of fission following incomplete fusion, and the relative detection probabilities for complete and incomplete fusion for each reaction. The data point for the ${ }^{232} \mathrm{Th}$ target shows a high multiplicity $\left(v_{\text {tot }}=14.5\right)$, reflecting the large positive fission $Q$ value, but also indicating that the fission coincidence geometry selected complete fusion rather cleanly for this reaction. This result is supported by the total neutron multiplicity measurement of Jahnke et al. ${ }^{41}$ for complete fusion of $220 \mathrm{MeV}{ }^{20} \mathrm{Ne}$ with ${ }^{238} \mathrm{U}$. It is worth noting that the current value of $v_{\text {tot }}$ for the $214 \mathrm{MeV}$ ${ }^{20} \mathrm{Ne}+{ }^{168} \mathrm{Er}$ reaction (9.5) is in excellent agreement with the value of 9.3 obtained $^{13}$ in the reaction of $220 \mathrm{MeV}$ ${ }^{20} \mathrm{Ne}$ with ${ }^{165} \mathrm{Ho}$, despite the different experimental geometry. The data of Ref. 17 for ${ }^{158} \mathrm{Er}$ do not fit in with the systematics of the other data, or with the expectations based on energy balance. It has already been shown ${ }^{20}$ that this indicates there is some discrepancy in these measurements, which also extends to the $v_{\text {pre }}$ values (see below).

\section{Pre-scission neutron multiplicities}

The pre-scission neutron multiplicities $v_{\text {pre }}$ measured in this work are shown in Fig. 3, together with experimental data from other sources ${ }^{9-20,42}$ for systems with similar masses or similar fissility $x$ (e.g., the ${ }^{158} \mathrm{Er}$ and ${ }^{168,170} \mathrm{Yb}$ systems). The data are shown as a function of the deduced excitation energy above the liquid drop model ground state of the compound nucleus, $E_{X}(\mathrm{CN})$. As in calculating $E_{X}(f), 20 \mathrm{MeV}$ was subtracted for each preequilibrium neutron. A further reduction in $E_{X}(\mathrm{CN})$ was made so that the measured total evaporated multiplicity 
agreed with that expected based on energy balance. In other words, the data shown in Fig. 2 were shifted in excitation energy so that they agreed with their respective mass curves, and this shift in excitation energy was applied to $E_{X}(\mathrm{CN})$. The shift applied showed a dependence both on the velocity at the fusion barrier and the fissility, as would be expected for processes resulting in incomplete transfer of excitation energy and angular momentum, and ranged from 0 to $15 \mathrm{MeV}$. For the present measurement for the ${ }^{158} \mathrm{Er}$ compound nucleus, no shift was required, implying that fission was only observed following complete fusion. This conclusion was also reached by the authors of Ref. 17 (for different reasons), so their data for ${ }^{158} \mathrm{Er}$ were also plotted with no shift.

The $v_{\text {pre }}$ data increase linearly with excitation energy, showing remarkable consistency. The only exceptions to this are the data of Ref. 17 for ${ }^{158} \mathrm{Er}$, which give $v_{\text {pre }}$ values considerably lower than those for ${ }^{168,170} \mathrm{Yb}$, and lower than the present data point for the same system. A full discussion of this discrepancy has already been pub-

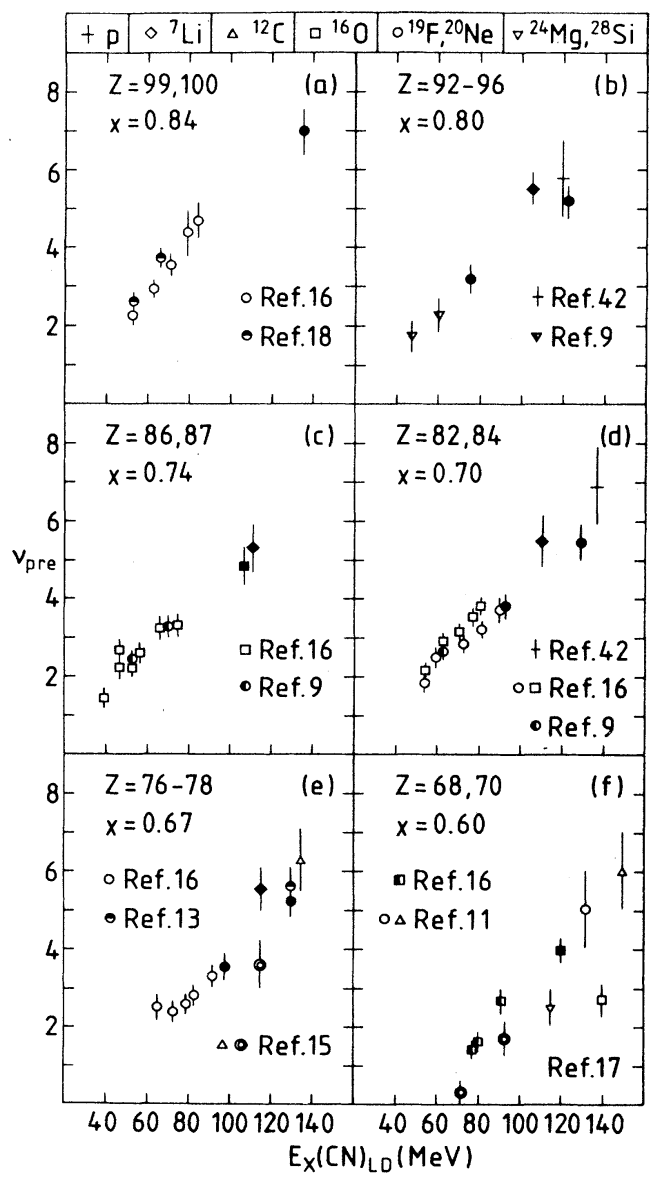

FIG. 3. Pre-scission neutron multiplicities for the present data, and for previous results, shown as a function of the excitation energy above the liquid drop ground state of the compound nuclei prior to fission. The typical fissility $x$ of the data in each panel is shown. Almost all the data show a remarkably consistent and smooth trend in both excitation energy and fissility. lished..$^{20}$ The only further point which will be made here is that in that work, ${ }^{20}$ a $v_{\text {pre }}$ value of $4.2 \pm 0.3$ was quoted for the present measurement for ${ }^{158} \mathrm{Er}$. This was obtained using in the analysis the TKE value from the systematics of $1966 .{ }^{32}$ Using the more recent formula, ${ }^{31}$ together with a slightly refined analysis results in the currently quoted value of $3.95 \pm 0.3$.

The prescission neutron multiplicity for a given $E_{X}(\mathrm{CN})$ increases as the fissility $x$ increases. This feature has been discussed in terms of the post-scission neutron multiplicity for measurements taken at lower excitation energy, ${ }^{9}$ and it seems that this reflects the increasing fission $Q$ value, offset by the decreasing rotational and deformation energies required in the fission process as the fissility increases.

The consistency of $v_{\text {pre }}$ data induced by projectiles ranging from protons to ${ }^{20} \mathrm{Ne}$ implies no gross change in the fission time scale as a function of angular momentum, although detailed calculations would have to be made to take into account the differences in binding energies and statistical model multiplicities which will affect a precise comparison. In order to come to quantitative conclusions regarding the angular momentum dependence of the fission time scale, it is necessary to have data with smaller uncertainties, and also information on the mean fissioning angular momentum for each reaction.

\section{Deduced fission time scales}

As was discussed in Sec. III C, time scales have been determined for the two extreme assumptions of saddlepoint (sp) emission and saddle-to-scission (ssc) emission. Calculations of the pre-scission neutron multiplicity as a function of time were made essentially as described in Ref. 16, using a modified version of the statistical model computer code ALERT. ${ }^{43}$ The effect of neutron emission

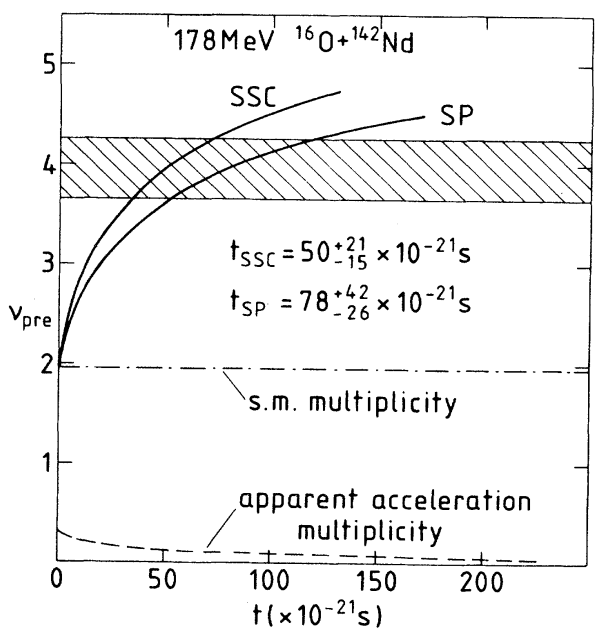

FIG. 4. For the reaction of $178 \mathrm{MeV}{ }^{16} \mathrm{O}+{ }^{142} \mathrm{Nd}$, the prescission neutron multiplicity $\left(v_{\text {pre }}\right)$ is shown as a function of the time allowed at the scission point (sp), or during the saddle-toscission transition (ssc). The shaded area represents the range of experimentally allowed values of $v_{\mathrm{pre}}$. The deduced values of $t_{\mathrm{sp}}$ and $t_{\mathrm{ssc}}$ are shown (see text). 
during the fission fragment acceleration time was accounted for as described there.

An example of the calculated relationship between $v_{\text {pre }}$ and time is shown in Fig. 4, for the extremes of sp and ssc emission. The statistical model calculations were made using a ratio of level density parameters $a_{f} / a_{n}=1.00$, and RLDM fission barrier scaling factors $k_{f}$ adjusted $^{16}$ to fit the measured fission probabilities at lower energies; $^{16,44-47} k_{f}$ values ranged from 0.82 to 1.05 as the fissility increased, and were within $\sim 5 \%$ of the Sierk barriers. It was found that use of the Sierk barriers makes only a very small change in the calculated statistical model multiplicities as would be expected (see Ref. 12). For the fissile nuclei ${ }^{229} \mathrm{~Np}$ and ${ }^{252} \mathrm{Fm}$, where no suitable fission probability data exists, $k_{f}$ was taken to be unity. This procedure of fitting the fission probability by scaling the barrier height could be questioned, as it does not explicitly account for any reduction in fission probability due to viscosity ${ }^{24}$ though the Kramers factor. ${ }^{48}$ It is felt that it is a reasonable approach, as will be further discussed in Sec. IV D. This is not however a problem

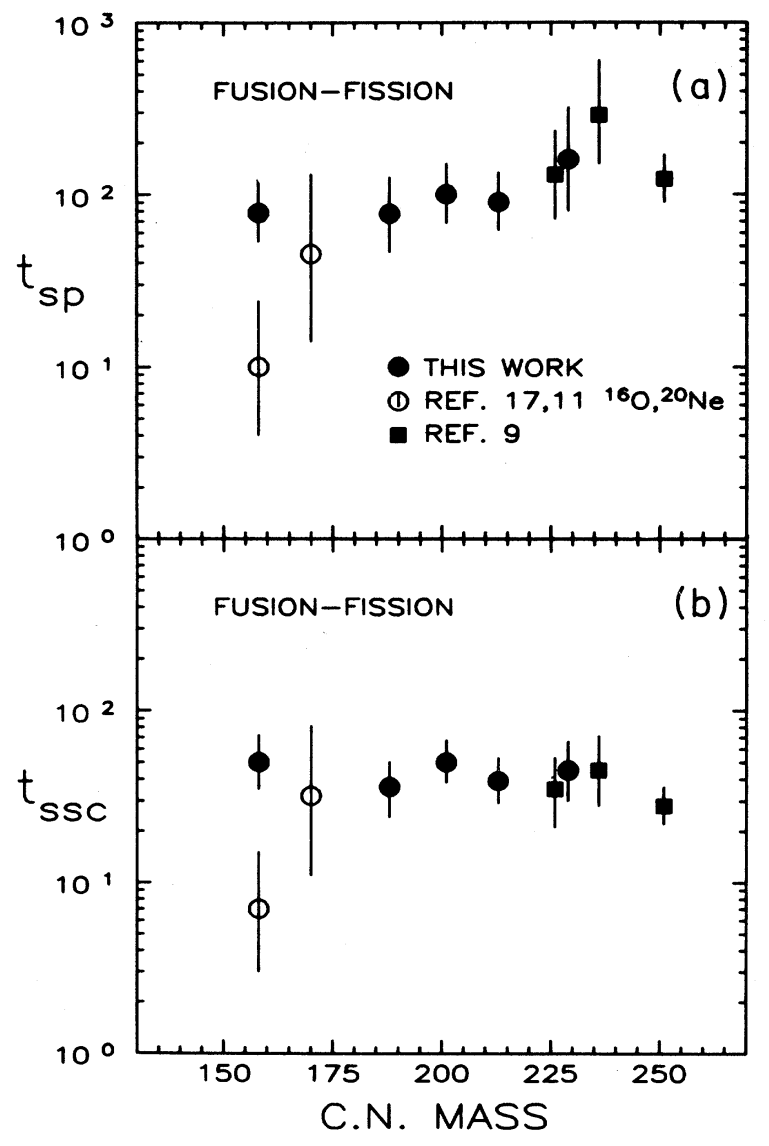

FIG. 5. Deduced values of $t_{\mathrm{sp}}$ (a) and $t_{\mathrm{ssc}}$ (b), in units of $10^{-21}$ $\mathrm{s}$, for the reactions classed as exclusively fusion-fission induced by ${ }^{16} \mathrm{O}$ and ${ }^{20} \mathrm{Ne}$, shown for convenience as a function of the compound system mass number. Also shown are the times deduced from $v_{\text {pre }}$ values given in the references indicated. All are for a level density parameter $a_{n}=A / 10$. where fast-fission is dominant, since the Kramers factor does not apply if there is no fission barrier. The use of $a_{f} / a_{n}=1.00$ is supported by the results of Ref. 19. A simultaneous unconstrained fit was performed there to both the fission probability data used here and prescission neutron multiplicities measured at lower energies where dynamical effects should be negligible. The value of $a_{f} / a_{n}$ was found to be strongly constrained by the $v_{\text {pre }}$ data, and was consistent with unity, within 0.02 .

The deduced fission time scales for the fusion-fission category of reactions are shown in Fig. 5, together with data from other publications ${ }^{11,15,17,18}$ for heavy-ion reactions without fast-fission. Panel $A$ gives the times calculated assuming $\mathrm{sp}$ emission $\left(t_{\mathrm{sp}}\right)$, shown for clarity as a function of the compound system mass, while panel $B$ shows those for ssc emission $\left(t_{\mathrm{ssc}}\right)$. The data point of Ref. 17 for ${ }^{158} \mathrm{Er}$ is that for the ${ }^{16} \mathrm{O}$ induced reaction which has been extensively quoted. ${ }^{49,50}$ It gives a time much shorter than all the other measurements. Apart from this point, the data show consistent behavior. For sp emission, the time scale increases by a factor of 1.5 as the fissility $x$ increases from 0.60 to 0.85 , while for ssc emission, it decreases by the same factor. Since the true

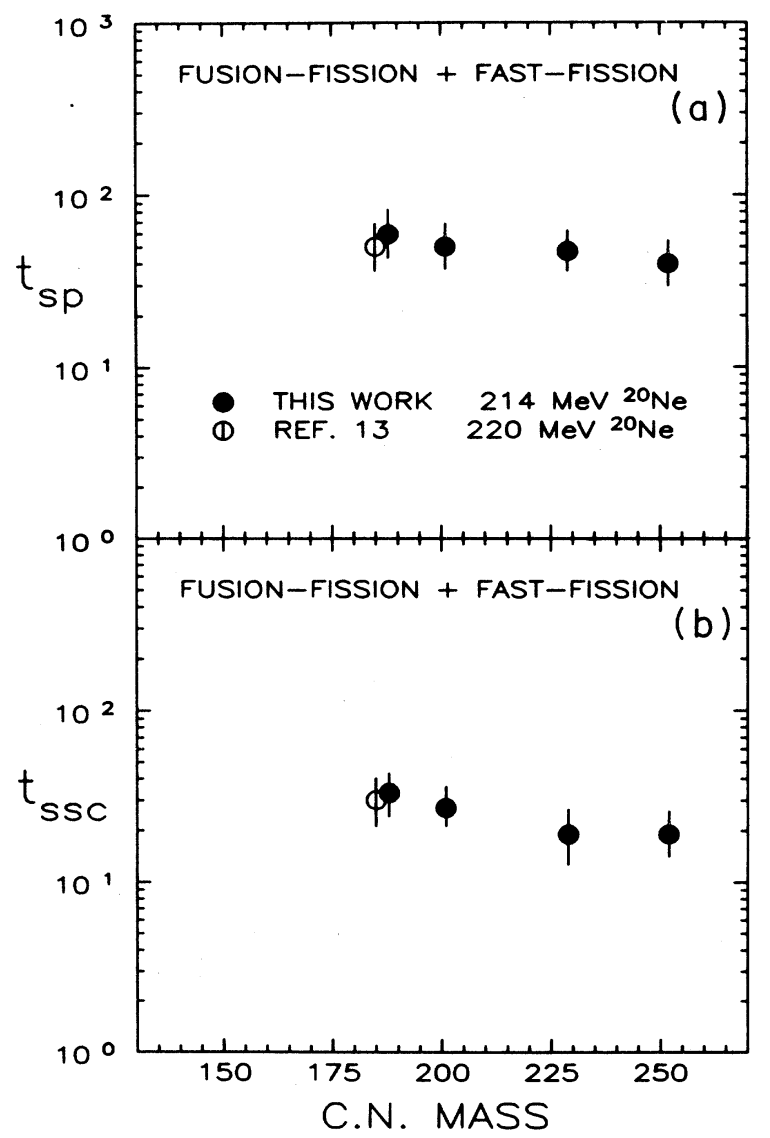

FIG. 6. As in Fig. 5, but for reactions where a fast-fission component is expected (see text and Table II). The times deduced are shorter than those in Fig. 5, the difference increasing with higher mass number (fissility) as might be expected (see text). 
situation must lie between these two extremes, it can be concluded that within error, the fusion-fission time scale is independent of fissility, at least for the range studied here. The only effect which could change this situation would be a variation of the level density parameter which was not proportional to mass.

Regarding the absolute times deduced, these depend on the level density parameter used, and would increase (decrease) by a factor of $\sim 2$ if $A / 8(A / 12)$ were used. The implications of such long time scales will be discussed in Sec. IV D, and the conclusions drawn will enable a simple interpretation of the lack of dependence of time scale on fissility.

The time scales deduced for those reactions which may have some fast-fission component according to the Bass model (see Table II) are shown in Fig. 6, both for sp and ssc emission. For the lighter systems, there is little reduction in time scale from that observed for the fusion-fission reactions, but for the heavier more fissile system, a clear reduction is observed, which for $\mathrm{sp}$ emission reaches about a factor of 3 . This difference in behavior can be attributed to two facts. The first is the reduction in angular momentum necessary to cause fast-fission as the fissility increases, shown in Table II. The second is the likely reduction by preequilibrium emission and incomplete fusion of the maximum angular momentum $l_{\text {crit }}$ (given in Table II) which can be brought into a system. For the lighter systems the velocity at the barrier is higher, and so these processes can be expected to be more likely, and may well result in no contribution from fastfission. These two effects would however cause an increase in the probability and proportion of fast-fission as the fissility increases.

The absolute time deduced for the reaction producing the heaviest system $\left({ }^{20} \mathrm{Ne}+{ }^{232} \mathrm{Th}\right)$ can be compared with those obtained from the deduced rotation angles in quasifission ${ }^{27}$ reactions between ${ }^{238} \mathrm{U}$ beams and targets ranging from ${ }^{16} \mathrm{O}$ to ${ }^{89} \mathrm{Y}$. From a comparison of the widths of the fission mass distributions, the reaction nearest to the present measurement (with a width of $60 \pm 5$ ) is that on the Al target, where a FWHM of $64 \pm 4$ was observed. For this reaction, only a lower limit to the reaction time, of $\sim 15 \times 10^{-21} \mathrm{~s}$ could be obtained. This value suggests that ssc emission for $a_{n}=A / 10$ represents a lower limit to the range of acceptable time scale calculations [see Fig. 6(b)], and thus that for the fusion-fission data [see Fig. 5(b)], the lower limit to the fusion-fission time scale is $30 \times 10^{-21} \mathrm{~s}$.

\section{B. ${ }^{7} \mathrm{Li}$ induced reactions}

For these data, several experimental uncertainties exist which make the deduction of time scales less reliable than for the data discussed above. The first uncertainty concerns the fission-neutron angular correlation data themselves. For the measurements on the ${ }^{197} \mathrm{Au}$ and ${ }^{209} \mathrm{Bi}$ targets, the $F$ detectors were not functional, so no momentum transfer selection could take place. Furthermore, it had been found that a fluctuating gating problem due to a faulty electronic unit affected all the TAC signals from the $f-F$ and $f$-neutron $(f-n)$ coincidences. When the neutron multiplicities were determined from the ratio of $f-F-n$ events to $f-F$ events, the effective dead time cancelled, but without the $F$ detectors, this dead time (which could not be measured otherwise) caused an unavoidable uncertainty in the two data points under discussion. From the dead times affecting previous runs with the $F$ detectors, the uncertainty for these two data points is estimated to be $\pm 12 \%$. As described in Sec. II, the ratio of $v_{\text {pre }} / \nu_{\text {post }}$ does not suffer from this uncertainty.

The measurements on the ${ }^{181} \mathrm{Ta}$ and ${ }^{232} \mathrm{Th}$ targets were made with $F$ detectors; for the latter target the $F$ detectors were placed to pick out only the highest momentum transfers. ${ }^{51}$ For the former target, the fission yield was rather low, and so rather poorer statistics were gathered.

The complex nature of the reaction mechanism for such a projectile and energy (see Ref. 51) makes interpretation of the measured $v_{\text {pre }}$ value subject to more uncertainty than the reactions induced by heavier ions. In fitting the neutron velocity spectra, it was found that a large preequilibrium neutron multiplicity was required to reproduce the high energy (velocity) tails of the spectra. It was assumed that the $v_{\text {pe }}$ yield was isotropic in azimuthal angle, and with $v_{\mathrm{pe}}=2.0$ it was possible to obtain good fits to the spectra when determining $v_{\text {pre }}$ and $v_{\text {post }}$.

The deduced total evaporated neutron multiplicities are shown in Fig. 2 as a function of $E_{X}(f)$. Rather good agreement with the calculations is found, although this may be fortuitous. The $v_{\text {pre }}$ values are shown in Fig. 3, and show qualitatively good agreement with the other systems. Statistical model calculations were made, however it was found that because of the lower angular momentum brought in, the deduced time scales were sensitive to the statistical model parameters used (for example the fusion cross section) thus no time scale can reliably be quoted without knowledge of more details of the reaction. In order to make reliable interpretations of pre-scission neutron multiplicities at such high bombarding energies per nucleon, more complete measurements should be made.

\section{Fission mass and TKE dependence of $\boldsymbol{v}_{\text {pre }}$}

In Table III, the width of the mass and TKE distributions are shown, together with the measured TKE (deduced assuming complete fusion). The widths are in good agreement with previous measurements, ${ }^{52}$ and the TKE results agree well with systematics ${ }^{31}$ after corrections for post-scission emission have been applied (assuming no pre-scission emission, as previously discussed). However, it has already been noted that during the measurement on the ${ }^{232} \mathrm{Th}$ target, an experimental problem with electron noise occurred. This, together with the intrinsic limits to the mass and TKE resolution due both to the solid angle of the $f$ detector and to neutron evaporation, gave a mass resolution estimated to be worse than 10 mass units. This has little effect on the total mass widths, which varied from 35 to $60 \mathrm{amu}$, but for mass bins of width 10-20 amu causes a considerable influence of the central mass bins (with more yield) on the peripheral ones. Thus a washing out of the variation of the measured neutron multiplicities may be expected. 
TABLE III. Results obtained for the measurements of fission mass and TKE distributions. Errors in measured quantities are estimated to be between $5 \%$ and $10 \%$.

\begin{tabular}{lcccccc}
\hline \hline Reaction & $\begin{array}{c}E_{\text {lab }}{ }^{\mathrm{a}} \\
(\mathrm{MeV})\end{array}$ & $\begin{array}{c}\text { TKE }^{\mathrm{b}} \\
(\mathbf{M e V})\end{array}$ & $\begin{array}{c}\text { FWHM } \\
\text { TKE } \\
(\mathbf{M e V})\end{array}$ & $\begin{array}{c}\text { FWHM } \\
\text { mass }\end{array}$ & $\begin{array}{c}\text { TKE }^{\mathrm{c}} \\
\text { corrected } \\
(\mathbf{M e V})\end{array}$ & $\begin{array}{c}\text { TKE } \\
\text { systematics } \\
(\mathbf{M e V})\end{array}$ \\
\hline $\mathrm{Ne}+\mathrm{Ta}$ & 149 & 141 & 30 & 35 & 147 & 144 \\
& 214 & 142 & 30 & 44 & 150 & 144 \\
$\mathrm{Ne}+\mathrm{Bi}$ & 149 & 169 & 38 & 41 & 175 & 175 \\
$\mathrm{Ne}+\mathrm{Th}$ & 214 & 183 & 48 & 60 & 194 & 195 \\
\hline \hline
\end{tabular}

${ }^{\mathrm{a}}$ Bombarding energy in center of target.

${ }^{b}$ Measured TKE.

${ }^{c}$ Measured TKE corrected for neutron emission as described in the text, to allow comparison with the TKE values expected from systematics shown in the next column (from Ref. 31).

Taking the measured TKE values to determine the mean fission fragment velocities in each bin, it was found that the dependence of $v_{\text {tot }}$ on TKE was not as steep as would be expected from calculations based on energy balance. However, by taking the TKE and mass values $30 \%$ closer to the central value, to account empirically for the poor resolution discussed above, the results shown in Fig. 7 were obtained. These exhibit the expected variation of $v_{\text {tot }}$ with TKE and with mass, determined from the calculations shown in Fig. 2 and knowledge of the appropriate $Q$ values.

The results indicate that $v_{\text {pre }}$, and thus the fission time scale, is within error independent of mass split, and also of TKE, except for the point at the lowest TKE. This

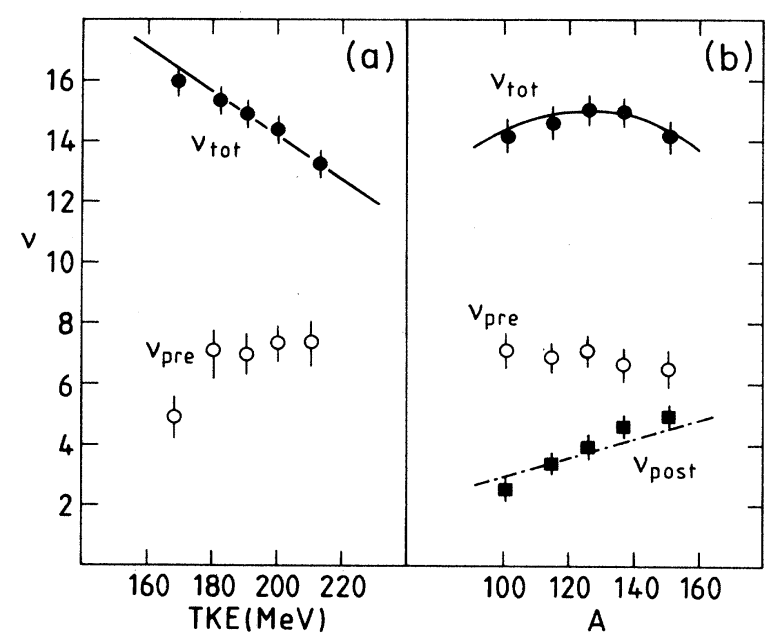

FIG. 7. Values of the total, pre- and post-scission neutron multiplicities as a function of the fission-fragment TKE (a), and mass (b), for the reaction of $215 \mathrm{MeV}^{20} \mathrm{Ne}+{ }^{232} \mathrm{Th}$. The estimated variation of $v_{\text {tot }}$ expected (calculated from the results of Fig. 2 and the appropriate $Q$ and TKE values) in each case is shown by the solid line, while in (b), the dot-dashed line shows $v_{\text {post }}$ proportional to fragment mass. The $v_{\text {pre }}$ values show no dependence on the fission fragment properties, with the possible exception of the point for the lowest TKE. point could only be increased to be in agreement with the others if the mean observed fragment mass was not half the scission mass, but one-third. From the mass and TKE correlations measured, this does not seem possible, however the rather poor resolution has been noted. In the $\mathrm{sp}$ emission picture this multiplicity corresponds to a time of $8 \times 10^{-21} \mathrm{~s}$, which is sufficient for the system at the saddle-point configuration to make more than one full rotation at an angular momentum of $70 \hbar$. Thus such a time is a physical possibility, and it would be interesting to see whether this effect is repeatable, or is an artifact of the experimental configuration. Until this is done, it is felt that speculation about the meaning of such a correlation of time scale and TKE, while entertaining, may be premature.

In the correlation of $v_{\text {pre }}$ with fragment mass, there is no evidence for a peak in multiplicity, and thus time, for more central mass splits, which might be expected if fast-fission and fusion-fission had mass distributions of distinctly different widths. The experimental data on mass widths argue against a large difference. For the reaction of $105 \mathrm{MeV}{ }^{19} \mathrm{~F}$ with the same target as in the present measurement, ${ }^{18}$ which should not result in fastfission, a width of $50 \pm 5$ amu was observed, not much narrower than at the present energy. The present data thus show that the fission process becomes $\sim 3$ times faster while the mass width increases only marginally in the transition from fusion-fission to fast-fission.

\section{Interpretation in terms of nuclear viscosity}

A model for the interpretation of fission time scales in terms of nuclear viscosity has already been used for the ${ }^{158} \mathrm{Er}$ nucleus, so the calculations made will also be utilized in this work. Some of the uncertainties in quantitative conclusions based on this model will be discussed. The theoretical framework for these calculations was developed by Grange, Weidenmüller, and others, ${ }^{22-24}$ based on pioneering work by Kramers. ${ }^{48}$ This model assumes that compound nuclei are formed initially close to the equilibrium deformation. Coupling between the particle degrees of freedom and the collective (fission) degrees of freedom is necessary to transform the initial population distribution to the quasistatic distribution extending to the saddle point which is implicit in the transition 
state model. However, this diffusion from the equilibrium configuration occurs over a transient time $\tau$, defined as the time during which the fission width rises from $0 \%$ to $90 \%$ of its quasistationary limit. If this time is greater than the neutron evaporation lifetime, this will affect the pre-scission neutron multiplicity. A second effect of viscosity is to reduce the current over the fission barrier (i.e., reduce the fission width). Because of fluctuations in the trajectory due to viscosity, nuclei which have passed the saddle point can return to the equilibrium pocket. Thus the value of $\tau_{\text {stat }}$ is increased. Viscosity will also increase the time taken to travel from the saddle point to the scission point $\tau_{\text {ssc }}$. It has been shown ${ }^{24}$ that the effective first-chance fission lifetime is approximately given by

$$
\tau_{f}=\tau_{\text {stat }}+0.5 \tau+\tau_{\text {ssc }}
$$

[for second-, third-chance fission, etc., the value of $\tau$ must be reduced to account for the time taken to emit the previous neutron(s)]. Thus, an increase in these times associated with fission will allow the emission of more neutrons than the statistical model calculations would suggest. A discussion of each of these effects will now be given.

Based on the conclusions from previous pre-scission neutron experiments, from the rapid dissipation of radial kinetic energy seen in deep inelastic reactions, and from the expectations for one-body viscosity, it is generally thought that nuclear viscosity should be large rather than very small. Thus in the following discussion, equations valid in this regime will be given. Viscosity is defined in terms of $\beta$, the ratio of the nuclear dissipation coefficient to the inertia. The potential energy surface (PES) in this model is approximated by harmonic oscillators (upright and inverted) whose frequencies are $\omega_{1}$ at the equilibrium deformation and $\omega_{0}$ at the saddle point (see Fig. 8).

The expression for the factor by which the fission width is reduced (the Kramers factor) is

$$
f_{K}=\left[\left(1+\gamma^{2}\right)^{1 / 2}-\gamma\right] \text {, }
$$

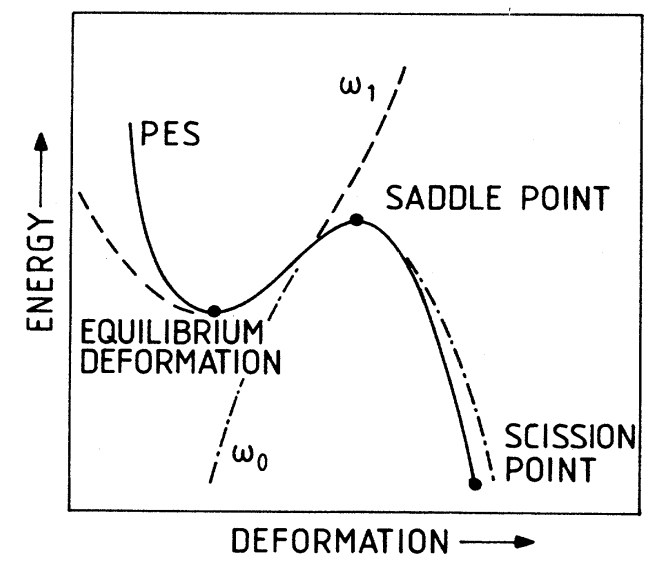

FIG. 8. Schematic representation of the way in which the true potential energy surface (PES) can be approximated in terms of harmonic oscillator potentials, as is done in the model of Refs. 22-25. where $\gamma=\beta / 2 \omega_{0}$. For overdamped motion ( $\gamma$ greater than 1) this factor can reduce the fission width substantially, and thus affect the statistical model value of $v_{\text {pre }}$. It is not yet clear how this factor should be treated. In Refs. 17 and 50 it was retained, and the fission probability was increased to its original (statistical model) value by increasing the value of the level density parameter at the saddle-point deformation $a_{f}$. Thus, the fission cross sections measured at lower energies are reproduced, but the statistical model value of $v_{\text {pre }}$ is severely reduced. ${ }^{49}$ Longer dynamical times are thus required to fit the data (compare Refs. 49 and 50, noting however that an excitation energy of $160 \mathrm{MeV}$ is used in Ref. 49, rather than $140 \mathrm{MeV}$ as in Ref. 50). The reduction in the statistical model value of $v_{\text {pre }}$ will extend to lower energies, and it will no longer be possible to fit with the statistical model data taken at low excitation energies [down to $40 \mathrm{MeV}$ (Refs. 9, 16, and 19)]. To explain a discrepancy here in terms of fission dynamics would require unreasonably long time scales. Since the fission probability data must still be fitted, our approach (as in Ref. 16) has been to multiply the RLDM fission barriers by a scaling factor $k_{f}$ to fit the fission probability data for the reactions studied, or for similar ones. ${ }^{16,44}$ Thus, the effect of the Kramers factor, the reduction of the fission barrier with temperature, and any changes in viscosity with temperature (which are all linked together in a complex manner and may cancel to some extent, but which we feel cannot yet be calculated quantitatively) are focused onto $k_{f}$. Though not fully satisfactory, this procedure does not require the large values of the ratio $a_{f} / a_{n}$ which the current $v_{\text {pre }}$ data would require (greater than 1.2), in conflict with the $v_{\text {pre }}$ results at lower bombarding energies, where the effect of the dynamical fission time scale should be small, and where values of $a_{f} / a_{n}$ close to 1.00 are required. ${ }^{16,19}$ There are also theoretical predictions that $a_{f} / a_{n}$ should be only slightly larger than unity ${ }^{53}$ (typically $\sim 1.04$, reducing to 1.00 as the fission barrier approaches zero). These differences in approach between this work and Ref. 17 mean that the difference in the deduced viscosity will not be as large as the difference in time scales (see Fig. 5) might suggest.

Turning to the fission delay time $\tau$, it can be approximately expressed as

$$
\tau=\frac{\beta}{2 \omega_{X}^{2}} \ln \left\{10 E_{f} / T\right\},
$$

where $E_{f}$ is the fission barrier height and $T$ is the temperature. The parameter $\omega_{X}$ is an oscillator frequency related to $\omega_{1}$, but modified to give the same results as a more sophisticated treatment. ${ }^{24}$ In the absence of such information, $\omega_{1}$ will be used, as in Refs. 23 and 54. This equation is valid for overdamped motion ( $\gamma$ greater than 1). For a given fission barrier height, the value of $\omega_{1}$ required to approximate the potential energy surface in the equilibrium pocket will (to first order) be inversely proportional to the distance from the minimum in that pocket to the saddle point. Thus the equation predicts that for a given $\beta$, the transient time is proportional to the square of the distance which must be traveled. This is exactly 
what would be expected in the case of a random walk (Brownian motion). The direct dependence on fission barrier height is rather small. However, if $E_{f}$ is doubled, fixing all other variables, the required value of $\omega_{1}$ is increased (to first order) by $\sqrt{2}$, and thus $\tau$ is halved (the logarithmic term increases $\tau$ only slightly). This is not what would be expected intuitively; an increase of barrier height should increase $\tau$. This may be partly due to the use of $\omega_{1}$ rather than $\omega_{X}$, but may also reflect the limitation of characterizing the PES in terms of two harmonic oscillators.

As the fission barrier is reduced, and $E_{f} / T$ becomes less than unity, this equation loses its validity, as the whole fission process is expected ${ }^{24,54}$ to have a transient nature, with no quasistationary condition to approach. In this regime, a different expression has been derived for the transient time to reach the scission point ${ }^{54}$

$$
\tau_{\mathrm{ssc}}=-\frac{1}{2 a_{2}} \ln \left[\frac{a_{2} \beta}{\epsilon\left(a_{2}-\beta\right)}\right],
$$

where $\epsilon=\beta T / \mu, a_{2}=\beta / 2-\omega_{z}$, and $\omega_{z}^{2}=\beta^{2} / 4+\omega_{0}^{2} ; \mu$ is the reduced mass, taken to be constant. For $\gamma$ greater than 2, this can be approximated as

$$
\tau_{\mathrm{ssc}}=\frac{\beta}{2 \omega_{0}^{2}} \ln \left[\frac{\omega_{0}^{2} \mu}{\beta^{2} T}\right) .
$$

This shows the same characteristic as Eq. (2), the time depending on the square of the distance to be traveled. Other expressions purely for the saddle-to-scission time ${ }^{24,54}$ have been derived, and give the same characteristic dependence on $\omega_{0}$, and similar times. A useful observation, ${ }^{55}$ valid for all values of $\gamma$, is that the saddle-toscission time is increased over that for no viscosity $\left(\tau_{\mathrm{ssc}}^{0}\right)$ as shown below

$$
\tau_{\mathrm{ssc}}=\left[\left(1+\gamma^{2}\right)^{1 / 2}+\gamma\right] \tau_{\mathrm{ssc}}^{0}
$$

In conjunction with hydrodynamical calculations of the saddle-to-scission time for no viscosity, this expression has been used in the interpretation of the previous results for ${ }^{158} \mathrm{Er}$. Using Eqs. (2) and (5), the relationships between $\tau, \tau_{\text {ssc }}$, and $\beta$ previously calculated can be extrapolated to allow a value of $\beta$ to be determined for the present measurement. From Eq. (1), the calculated time which should be compared with that deduced experimentally is $\tau / 2+\tau_{\text {ssc }}$. This is the quantity which has been called the fission time scale. Shown in Fig. 9 is the variation of this quantity, and also of $\tau, \tau_{\text {ssc }}$ for an angular momentum of $65 \hbar$. The acceptable range of fission time scales deduced from the data is enclosed by the dotdashed lines, this range includes the uncertainty of sp or ssc emission. The viscosity is very large, within the range $\beta=14 \times 10^{21} \mathrm{~s}^{-1}$ to $51 \times 10^{21} \mathrm{~s}^{-1}$. Since typical values of $\omega_{0}$ and $\omega_{1}$ are $\sim 1 \times 10^{21} \mathrm{~s}^{-1}$, it can be firmly concluded within this model, the motion is highly overdamped. It has already been noted that this model predicts larger values of $\tau$ for lower barriers, which is contrary to intuition. If the temperature-induced reduction of the fission barrier ${ }^{56,57}$ is considered, this feature of the model would lead to smaller required values of $\beta$, but $\omega_{0}$ would also be

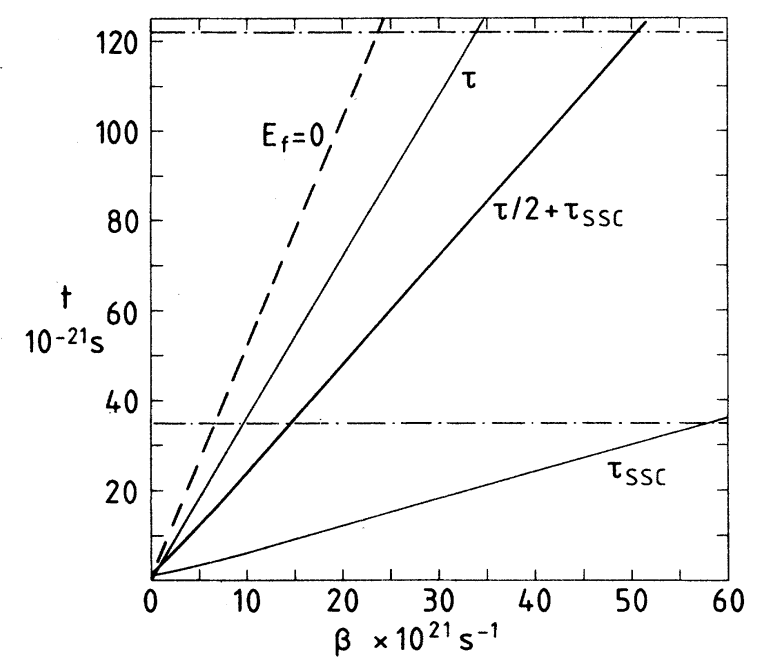

FIG. 9. The calculated fission delay time $\tau$, the saddle-toscission time $\tau_{\mathrm{ssc}}$ and the fission time scale $\left(\tau / 2+\tau_{\mathrm{ssc}}\right)$ as a function of the nuclear viscosity coefficient $\beta$, for the nucleus ${ }^{158} \mathrm{Er}$ at angular momentum $65 \hbar$, extrapolated from the results shown in Ref. 50, using equations valid for overdamped motion (see text) which do not describe the rapid increase in time scale for very low viscosity. Also shown (by the dashed line labeled $E_{f}=0$ ) is the time to reach the scission point if the barrier is reduced to zero. The dot-dashed lines indicate the range of times allowed by the experimental datum for this reaction.

reduced, and the degree of overdamping would not be reduced so much. If however it is assumed that the fission barrier is reduced to zero, Eq. (4) becomes appropriate to calculate the transient time to reach the scission point. Calculated values for ${ }^{158} \mathrm{Er}$ are shown in Fig. 9 by the dashed line labeled $E_{f}=0$. The acceptable range for $\beta$ is between 8 and $22 \times 10^{21} \mathrm{~s}^{-1}$, and with $\omega_{1} \sim 0.65 \times 10^{21}$ $\mathrm{s}^{-1}$, a strongly overdamped situation is still required.

In conclusion, uncertainties in the input parameters and the model itself make the quotation of a firm value of $\beta$ of questionable value, particularly in view of the variation of the friction coefficient and damping factor with deformation predicted in the model of Yamaji et al. ${ }^{58}$ Nevertheless, it can be confidently stated that the dominant characteristic of motion in the fission direction is strong overdamping. This has two immediate consequences. The first is that the kinetic energy at scission should be low, and thus the scission configuration should be almost independent of fissility, i.e., the fragment center-to-center distance should scale almost as $A^{1 / 3}$ (see, for example, calculations based on the one-body dissipation mechanism in Ref. 7). The second, and more important consequence is that by definition, the motion from the equilibrium deformation almost to scission will be dominated by the fluctuations associated with the large viscosity. Unless the values of $\omega$ required to fit the PES are so large that overdamping is no longer the case, the motion towards scission will be largely independent of the PES, and should just depend on the distance to be 
traveled, as implied in Eqs. (2) and (4). These two facts then lead inevitably to the conclusion that the fission time scale should be almost independent of fissility. This is exactly what has been found experimentally in this work.

The experimental observation of long fission time scales which are independent of fissility are thus consistent. They suggest that despite the limitations of the model used to deduce values of the viscosity coefficient $\beta$, the interpretation of fission dynamics in terms of a diffusion process, or random walk, is correct, and may be the essential physical ingredient required for a clearer understanding not only of pre-scission neutron multiplicities, but also of many other properties in heavy-ion induced fission, such as widths of fission mass and TKE distributions, and fission angular distributions.

\section{SUMMARY}

A new and comprehensive set of pre-scission neutron multiplicity data for ${ }^{16} \mathrm{O}$ and ${ }^{20} \mathrm{Ne}$ induced reactions have been presented, spanning a fissility range of 0.60 to 0.85 . They include measurements both for fusion-fission reactions only, and for reactions in which fast-fission is expected.

It has been shown that a previous measurement of neutron multiplicities for reactions leading to fission following formation of the compound nucleus ${ }^{158} \mathrm{Er}$ give $v_{\text {tot }}$ results inconsistent with other data and with expectations based on energy balance, and $v_{\text {pre values lower than }}$ might be expected from systematics. A new measurement was made for the reaction of $178 \mathrm{MeV}{ }^{16} \mathrm{O}+{ }^{142} \mathrm{Nd}$ which resulted in a consistent value of $v_{\text {tot }}$, and a $v_{\text {pre }}$ value of $3.95 \pm 0.3$, considerably higher than the previous result for a $207 \mathrm{MeV}{ }^{16} \mathrm{O}$ beam of $2.7 \pm 0.4$. This new result leads to a much longer dynamical fission time scale and higher viscosity for ${ }^{158} \mathrm{Er}$ than was concluded from the previous measurement.

A method has been used to reliably deduce fission time scales from $v_{\text {pre }}$ data even when complete fusion is not expected, by making use of the $v_{\text {tot }}$ result to determine the initial excitation energy. This relies upon experimental data with small systematic errors. The absence of such errors has been checked by comparing the values of $v_{\text {tot }}$ with systematics and expectations based on energy balance for the reactions at lower energies where complete fusion should be dominant.

The $v_{\text {pre }}$ data have been transformed to time scales for the minimum and maximum possible mean excitation energies during fission; respectively, neutron emission from the saddle-point ( $\mathrm{sp}$ emission) and during the saddle-toscission transition (ssc emission). For these extreme assumptions, the variation of the deduced fusion-fission time scale with compound nucleus fissility has been investigated. It was found that the fission time scale showed an increase or decrease with fissility by a factor of 1.5 for $\mathrm{sp}$ and ssc emission, respectively. Since the true situation will lie between the extremes, it can be concluded that the fusion-fission time scale is independent of fissility within this uncertainty. The absolute times deduced depend on whether sp or ssc emission is assumed, and on the level density parameter. From a comparison of the fast-fission data with quasifission time scales deduced from rotation angles, a minimum fusion-fission time scale of $30 \times 10^{-21}$ s has been estimated.

Measurements for ${ }^{7} \mathrm{Li}$ induced fission reactions indicate a similar time scale as for fission induced by heavier ions, but the data and interpretation are subject to more uncertainties.

The dependence of $v_{\text {pre }}$ on the fission fragment mass, and on the TKE, was measured for the reaction of 215 $\mathrm{MeV}{ }^{20} \mathrm{Ne}$ on ${ }^{232} \mathrm{Th}$. No variation was found except for the lowest values of the TKE, where a reduction in multiplicity was observed. It would be interesting to see whether this effect is repeatable, or is an artifact of the experimental configuration. The lack of correlation of $v_{\text {pre }}$ with mass is consistent with the small increase in mass width observed in the transition from fusion-fission to fast-fission.

For those systems where a significant fast-fission component was expected, a reduction in time scale was observed. For the lighter systems, there is little or no reduction in time scale, but for the heavier more fissile systems, a substantial reduction is observed. This can be explained by the reduction in angular momentum necessary to cause fast-fission as the fissility increases, and the likely reduction of the maximum angular momentum which can be brought into a system caused by the processes of preequilibrium emission and incomplete fusion. From the data for the heaviest system, where most of the fission yield should be fast-fission, it has been shown to be up to three times faster than fusion-fission, but is slower than a typical quasifission reaction, as is also indicated by the width of the mass distribution.

A model developed by Grange, Weidenmüller, and others was used to interpret the long fission time scales deduced. It was concluded that the motion towards scission is strongly overdamped. A picture of fission dynamics in terms of a slow diffusion towards the scission point is implied, where the time required for changing the deformation is not greatly influenced by the detailed shape of the rather flat potential energy surface, except very close to the scission point. Pre-scission kinetic energies will thus be low, and the scission configuration will be compact, and not very dependent on fissility. These facts together indicate that the fission time scale should be almost independent of fissility, as has been observed in this work.

\section{ACKNOWLEDGMENTS.}

The authors wish to thank the cyclotron staff for providing a wide range of beams in a short time, Prof. $\mathbf{H}$. Baba and Dr. T. Saito for assistance in obtaining a ${ }^{252} \mathrm{Cf}$ source, Dr. M. Fujiwara, Dr. H. Ikezoe, Dr. J. R. Leigh, and Dr. T. Saito for providing targets, and Dr. S. Yamaji for his critical comments on the manuscript of this paper. One of us (D.J.H.) acknowledges the support of the Japan Society for the Promotion of Science during his year at the Research Center for Nuclear Physics (RCNP), and would like to thank Dr. D. Hilscher and Dr. H. Rossner for many stimulating discussions and help at HMI. This experiment was performed under RCNP Program No. 24A22. 
${ }^{*}$ Present address: Hahn-Meitner-Institut, D-1000 Berlin 39, Federal Republic of Germany.

${ }^{\dagger}$ Present address: Department of Chemistry, Faculty of Science, Nagoya University, Nagoya 464, Japan.

${ }_{1}^{1}$ J. R. Nix and A. J. Sierk, Adriatic International Conference on Nuclear Physics-Frontiers of Heavy-Ion Physics, Dubrovnik, Yugoslavia 1987, edited by R. Caplar, N. Cindro, and M. Korolija [Fizika 19, Suppl. 1, 333 (1987)].

${ }^{2}$ P. Bonche, S. E. Koonin, and J. W. Negele, Phys. Rev. C 13, 1226 (1976).

${ }^{3}$ W. J. Swiatecki, Nucl. Phys. A428, 199c (1984) and references therein.

${ }^{4}$ J. R. Nix and A. J. Sierk, Phys. Rev. C 21, 396 (1980), and references therein.

${ }^{5}$ P. Fröbrich, Phys. Rep. 116C, 337 (1984).

${ }^{6}$ W. Nörenberg, Nucl. Phys. A428, 177c (1984).

${ }^{7}$ K. T. R. Davies, A. J. Sierk, and J. R. Nix, Phys. Rev. C 13, 2385 (1976); J. R. Nix and A. J. Sierk, International Conference on Nuclear Physics on The Golden Jubilee of the Indian National Science Academy, Bombay, India, 1984, edited by B. K. Jain and B. C. Sinha (World Scientific, Singapore, 1985), p. 365.

${ }^{8}$ J. Toke, R. Bock, G. X. Dai, A. Gobbi, S. Gralla, K. D. Hildenbrand, J. Kuzminski, W. J. F. Müller, A. Olmi, H. Stelzer, B. B. Back, and S. Bjornholm, Nucl. Phys. A440, 327 (1985).

${ }^{9}$ D. J. Hinde, J. J. M. Bokhorst, J. R. Leigh, and J. O. Newton (unpublished)

${ }^{10}$ For example, L. C. Vaz, D. Logan, E. Duek, J. M. Alexander, M. F. Rivet, M. S. Zisman, M. Kaplan, and J. W. Ball, Z. Phys. A 315, 169 (1984); N. N. Ajitanand, J. M. Alexander, H. Delagrange, E. Duek, D. O. Eriksen, D. Guerreau, M. Kaplan, M. Kildir, L. Kowalski, R. Lacey, D. Logan, D. J. Moses, G. F. Peaslee, L. C. Vaz, and M. S. Zisman, ibid. 316, 169 (1984); L. Schad, H. Ho, G.-Y. Fan, B. Lindl, A. Pfoh, R. Wolski, and J. P. Wurm, ibid. 318, 179 (1984), and references therein.

${ }^{11}$ A Gavron, J. R. Beene, B. Cheynis, R. L. Ferguson, F. E. Obenshain, F. Plasil, G. R. Young, G. A. Petitt, R. Jaaskelainen, D. G. Sarantites, and C. F. Maguire, Phys. Rev. Lett. 47, 1255 (1981); 48, 835(E) (1982).

${ }^{12}$ D. Ward, R. J. Charity, D. J. Hinde, J. R. Leigh, and J. O. Newton, Nucl. Phys. A424, 189 (1983).

${ }^{13}$ D. Hilscher, E. Holub, U. Jahnke, H. Orf, and H. Rossner, in Proceedings of the Third Adriatic Europhysics Study Conference, Hvar, Yugoslavia, 1981, edited by N. Cindro, R. A. Ricci, and W. Greiner (North-Holland, Amsterdam, 1981), p. 225; E. Holub, D. Hilscher, G. Ingold, U. Jahnke, H. Orf, and H. Rossner, Phys. Rev. C 28, 252 (1983).

${ }^{14}$ D. J. Hinde, R. J. Charity, G. S. Foote, J. R. Leigh, J. O. Newton, S. Ogaza, and A. Chatterjee, Phys. Rev. Lett. 52, 986 (1984); 53, 2275(E) (1984).

${ }^{15}$ W. P. Zank, D. Hilscher, G. Ingold, U. Jahnke, M. Lehmann, and H. Rossner, Phys. Rev. C 33, 519 (1986).

${ }^{16}$ D. J. Hinde, R. J. Charity, G. S. Foote, J. R. Leigh, J. O. Newton, S. Ogaza, and A. Chatterjee, Nucl. Phys. A452, 550 (1986).

${ }^{17}$ A. Gavron, A. Gayer, J. Boissevain, H. C. Britt, T. C. Awes, J. R. Beene, B. Cheynis, D. Drain, R. L. Ferguson, F. E. Obenshain, F. Plasil, G. R. Young, G. A. Petitt, and C. Butler, Phys. Rev. C 35, 579 (1987).

${ }^{18}$ D. J. Hinde, J. R. Leigh, J. J. M. Bokhorst, J. O. Newton, R. L. Walsh, and J. E. Boldeman, Nucl. Phys. A472, 318 (1987).

${ }^{19}$ J. O. Newton, D. J. Hinde, R. J. Charity, J. R. Leigh, J. J. M. Bokhorst, A. Chatterjee, G. S. Foote, and S. Ogaza, Nucl.
Phys. A483, 126 (1988).

${ }^{20}$ D. J. Hinde, H. Ogata, M. Tanaka, T. Shimoda, N. Takahashi, A. Shinohara, S. Wakamatsu, K. Katori, and H. Okamura, Phys. Rev. C 37, 2923 (1988).

${ }^{21}$ N. Carjan, A. J. Sierk, and J. R. Nix, Nucl. Phys. A452, 381 (1986).

22P. Grange and H. A. Weidenmüller, Phys. Lett. 96B, 26 (1980).

${ }^{23}$ H. A. Weidenmüller and Zhang Jing-Shang, Phys. Rev. C 29, 879 (1984).

${ }^{24}$ K. H. Bhatt, P. Grange, and B. Hiller, Phys. Rev. C 33, 954 (1986).

${ }^{25}$ P. Grange, Li Jun-Quing, and H. A. Weidenmüller, Phys. Rev. C 27, 2063 (1983).

${ }^{26}$ C. Gregoire, C. Ngo, E. Tomasi, B. Remaud, and F. Scheuter, Nucl. Phys. A387, 37c (1982), and references therein.

${ }^{27}$ W. Q. Shen, J. Albinski, A. Gobbi, S. Gralla, K. D. Hildenbrand, N. Herrmann, J. Kuzminski, W. J. F. Müller, H. Stelzer, J. Toke, B. B. Back, S. Bjornholm, and S. P. Sorensen, Phys. Rev. C 36, 115 (1987).

${ }^{28}$ T. Sikkeland, E. L. Haines, and V. E. Viola, Jr., Phys. Rev. 125, 1350 (1962).

${ }^{29}$ S. B. Kaufman, E. P. Steinberg, B. D. Wilkins, J. Unik, and A. J. Gorski, Nucl. Instrum. Methods 115, 47 (1974).

30J. Grundl and C. Eisenhauer, Natl. Bur. Stds. Pub. NBS-493 (1977).

${ }^{31}$ V. E. Viola, K. Kwiatkowski, and M. Walker, Phys. Rev. C 31, 1550 (1985).

${ }^{32}$ V. E. Viola, Jr., Nucl. Data Tables A1, 391 (1966).

${ }^{33}$ A. Gavron, J. R. Beene, B. Cheynis, R. L. Ferguson, F. E. Obenshain, F. Plasil, G. R. Young, G. A. Petitt, C. F. Maguire, D. G. Sarantites, M. Jaaskelainen, and K. Geoffroy-Young, Phys. Rev. C 27, 450 (1983).

${ }^{34}$ E. Holub, D. Hilscher, G. Ingold, U. Jahnke, H. Orf, H. Rossner, W. P. Zank, W. U. Schroder, H. Gemmeke, K. Keller, L. Lassen, and W. Lucking, Phys. Rev. C 33, 143 (1986).

${ }^{35}$ S. Cohen, F. Plasil, and W. J. Swiatecki, Ann. Phys. (N.Y.) 82, 557 (1974).

${ }^{36}$ A. J. Sierk, Phys. Rev. C 33, 2039 (1986).

${ }^{37}$ S. Henss, A. Ruckelhausen, R. D. Fischer, W. Kühn, V. Metag, R. Novotny, R. V. F. Janssens, T. L. Khoo, D. Habs, D. Schwalm, D. Freeman, G. Duchene, B. Haas, F. Haas, S. Hlavac, and R. S. Simon, Phys. Rev. Lett. 60, 11 (1988).

${ }^{38}$ M. Thoennessen, D. R. Chakrabarty, M. G. Herman, R. Butsch, and P. Paul, Phys. Rev. Lett. 59, 2860 (1987).

${ }^{39}$ M. Blann and B. Remington, Phys. Rev. C 37, 2231 (1988).

${ }^{40}$ R. Bass, Phys. Rev. Lett. 39, 265 (1977).

${ }^{41}$ U. Jahnke, G. Ingold, D. Hilscher, M. Lehmann, E. Schwinn, and P. Zank, Phys. Rev. Lett. 57, 190 (1986).

${ }^{42}$ E. Cheifetz, Z. Fraenkel, J. Galin, M. Lefort, J. Peter, and X. Tarrago, Phys. Rev. C 2, 256 (1970).

${ }^{43}$ M. Blann and T. A. Komoto, Lawrence Livermore Laboratory Report No. UICD 19390.

${ }^{44}$ R. J. Charity, J. R. Leigh, J. J. M. Bokhorst, A. Chatterjee, G. S. Foote, D. J. Hinde, J. O. Newton, S. Ogaza, and D. Ward, Nucl. Phys. A457, 441 (1986).

${ }^{45}$ A. Gavron, J. Boissevain, H. C. Britt, K. Eskola, P. Eskola, M. M. Fowler, H. Ohm, J. B. Wilhelmy, T. C. Awes, R. L. Ferguson, F. E. Obenshain, F. Plasil, G. R. Young, and S. Wald, Phys. Rev. C 30, 1550 (1984).

${ }^{46}$ M. L. Halbert, R. A. Dayras, R. L. Ferguson, F. Plasil, and D. G. Sarantites, Phys. Rev. C 17, 155 (1978).

${ }^{47}$ A. M. Zebelman, L. Kowalski, J. Miller, K. Beg, Y. Eyal, G. Jaffe, A. Kandil, and D. Logan, Phys. Rev. C 10, 200 (1974). 
${ }^{48}$ H. A. Kramers, Physica 7, 284 (1940).

${ }^{49}$ S. Hassani and P. Grange, Z. Phys. A 325, 95 (1986).

${ }^{50}$ P. Grange, S. Hassani, H. A. Weidenmüller, A. Gavron, J. R. Nix, and A. J. Sierk, Phys. Rev. C 34, 209 (1986), and references therein.

${ }^{51}$ M. Fatyga, H. J. Karwowski, K. Kwiatkowski, L. Nowicki, V. E. Viola, and K. Hicks, Phys. Rev. C 35, 568 (1987).

${ }^{52}$ C. C. Sahm, H. Schulte, D. Vermeulen, J. Keller, H.-G. Clerc, K.-H. Schmidt, F. Hessberger, and G. Munzenberg, Z. Phys. A 297, 241 (1980).
${ }^{53}$ J. Toke and W. J. Swiatecki, Nucl. Phys. A372, 141 (1981).

${ }^{54}$ P. Grange, Nucl. Phys. A428, 37c (1984).

${ }^{55}$ H. Hofmann and J. R. Nix, Phys. Lett. 122B, 117 (1983).

${ }^{56}$ J. Bartel and P. Quentin, Phys. Lett. 152, 29 (1985), and references therein.

${ }^{57}$ H. Delagrange, C. Gregoire, F. Scheuter, and Y. Abe, Z. Phys. A 323, 437 (1986).

${ }^{58}$ S. Yamaji, H. Hofmann, and R. Samhammer, Nucl. Phys. A475, 487 (1988). 